\title{
Orthogonal flexible Rydberg aggregates
}

\author{
K. Leonhardt, ${ }^{1, *}$ S. Wüster, ${ }^{1,2}$ and J. M. Rost ${ }^{1}$ \\ ${ }^{1}$ Max Planck Institute for the Physics of Complex Systems, Nöthnitzer Strasse 38, D-01187 Dresden, Germany \\ ${ }^{2}$ Department of Physics, Bilkent University, Ankara 06800, Turkey
}

(Received 20 November 2015; published 16 February 2016)

\begin{abstract}
We study the link between atomic motion and exciton transport in flexible Rydberg aggregates, assemblies of highly excited light alkali-metal atoms, for which motion due to dipole-dipole interaction becomes relevant. In two one-dimensional atom chains crossing at a right angle adiabatic exciton transport is affected by a conical intersection of excitonic energy surfaces, which induces controllable nonadiabatic effects. A joint exciton-motion pulse that is initially governed by a single energy surface is coherently split into two modes after crossing the intersection. The modes induce strongly different atomic motion, leading to clear signatures of nonadiabatic effects in atomic density profiles. We have shown how this scenario can be exploited as an exciton switch, controlling direction and coherence properties of the joint pulse on the second of the chains [K. Leonhardt $e t$ al., Phys. Rev. Lett. 113, 223001 (2014)]. In this article we discuss the underlying complex dynamics in detail, characterize the switch, and derive our isotropic interaction model from a realistic anisotropic one with the addition of a magnetic bias field.
\end{abstract}

DOI: 10.1103/PhysRevA.93.022708

\section{INTRODUCTION}

Over the last decade fundamental phenomena in many-body Rydberg systems have been discovered, from dipole blockade [1-4] and antiblockade [5,6] over long-range molecules $[7,8]$ to excitonic dynamics due to resonant dipole-dipole interactions [9-18]. Underlying all of these are strong longrange interactions [19-23], which also make Rydberg atoms promising for quantum computing [24], quantum simulators $[25,26]$, and model systems for biological processes [18,27].

It also became apparent that the interplay of atomic motion and excitonic dynamics [28] yields entanglement transport $[10,11]$ and conical intersections (CIs) [12]. The latter play a crucial role in many quantum chemical processes, where they enable highly nonadiabatic dynamics on an ultrafast time scale [29]. They may also protect the DNA structure from damage by UV radiation [30]. In Ref. [31] we have explored how conical intersections affect the wave packets of atomic motion, which describe the entanglement transport and the associated energy and momentum transfer [10]. We have shown that the CI can split those wave packets, resulting in a superposition of two different excitation transfer processes. Since the relative weight of the processes after the splitting can be tuned through the system geometry and hence CI position, this gives rise to a sensitive switch for exciton transport properties.

The aforementioned intimate links between Rydberg atomic motion/exciton dynamics and biological as well as chemical energy transport processes [12,27] predestine ultracold Rydberg dynamics for quantum simulations and model system investigations in that context. These, however, require a deeper understanding of moving Rydberg systems. Their underlying exciton spectra as a function of time we will discuss here in detail. We also provide a parameter space survey for the operation of the conical intersection switch. Our results are derived from a model of isotropic dipole-dipole interactions. We finally show qualitatively similar features using the inherently anisotropic dipole-dipole interactions,

*karlo@pks.mpg.de where the anisotropy is suppressed through the application of an external magnetic field.

Dipole-dipole forces between atoms in two different Rydberg states $|s\rangle$ and $|p\rangle$ cause a strong interdependence of atomic motion and Rydberg state dynamics [9]. On a homogeneous chain of all but one atom in state $|s\rangle$, a single $|p\rangle$ excitation will delocalize forming a Frenkel exciton [28]. If the chain has a dislocation formed by two more closely spaced atoms, the exciton localizes on these and in a repulsive state causes a combined pulse of chain dislocation, excitation, and entanglement to propagate through the chain, akin to Newton's cradle [10]. In the scenario considered here, this combined pulse is directed towards a second chain orthogonal to the first, causing atoms to reach a configuration with a conical intersection in the exciton energy spectrum. The intersection causes nonadiabatic effects, triggering two different modes of pulse propagation on the second chain. The basic mechanism by which the conical intersection acts as a switch between these modes, can best be understood considering the essential subunit of two orthogonal atomic dimers.

The paper is organized as follows: In Sec. II, we discuss Rydberg aggregates and their numerical treatment. We then proceed in Sec. III A to describe a double Rydberg dimer, with two atoms freely moving on each of two orthogonal chains. We study the consequences of a conical intersection and characterize the dynamics on each of the two participating potential surfaces. Subsequently, in Sec. III B we extend this setup to a seven atom system, with three and four atoms on the two chains. In Sec. IV we highlight how the conical intersection can be functionalized as a switch controlling the transport dynamics on the second chain. Finally in Sec. V we examine the feasibility to realize our isotropic interaction model experimentally. The appendix contains technical details regarding the engineering of isotropic interactions and removal of spin degrees of freedom using a magnetic field.

\section{RYDBERG AGGREGATES}

We study a system of $N$ Rydberg atoms with masses $M$, all with the same principal quantum number $v$ which we restrict 

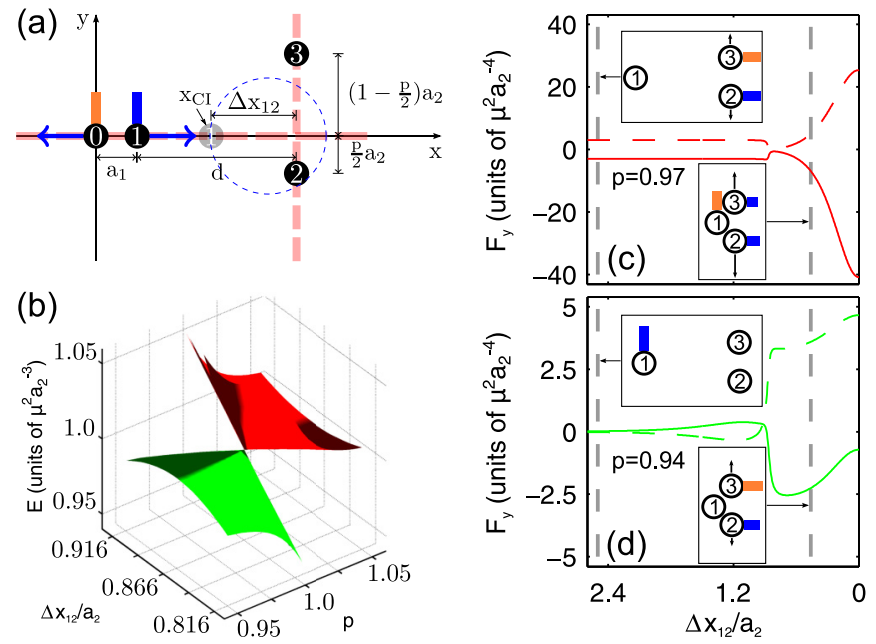

FIG. 1. (a) Orthogonal atom chains with one Rydberg dimer each. Atoms 0 and 1 initially share an excitation. Due to the ensuing repulsion (blue arrows) atom 1 reaches the conical intersection at $x_{\mathrm{CI}}$. The colored bars visualize the excitation amplitude on each atom $c_{n}=\left\langle\pi_{n} \mid \varphi_{\text {rep }}\right\rangle$, with orange $c_{n}>0$ and blue $c_{n}<0$. The origin of the coordinate system is set to the mean initial position of atom 0 . (b) The repulsive energy surface $U_{\text {rep }}$ (red) and adjacent surface $U_{\text {mid }}$ (green) of the trimer subunit (atoms 1, 2, and 3) near the CI. (c) and (d) Forces on atom 2 (solid lines) and atom 3 (dashed lines), for the repulsive surface [red, (c)] and adjacent surface [green, (d)]. The insets show atomic positions and the excitation distribution $c_{n}$ of exciton states and forces for the indicated values of $\Delta x_{12}$, which denotes the distance between atom 1 and the vertical chain. The parameter $p$ controls the degree of symmetry of the trimer, where $p=1$ corresponds to an isosceles trimer configuration. Figure reproduced from [31].

for the sake of clarity to the two cases $v=44$ and $v=80$. With $N_{x}$ of the atoms constrained on the $x$ axis, and $N_{y}$ on the $y$ axis their total number is $N=N_{x}+N_{y}$, for a simple example see Fig. 1(a). All atoms can move freely in only one dimension, with their positions described by the vector $\mathbf{R}=\left(\mathbf{R}_{1}, \ldots, \mathbf{R}_{n}\right)^{\mathrm{T}}$. The one-dimensional confinement could, for example, be realized by running laser fields and optical trapping of alkali-metal Rydberg atoms [32], or earth alkalimetal Rydberg atoms through their second valence electron [33]. Furthermore, we assume atoms prepared such that only one atom is in an angular momentum $p$ state, all the other atoms are in angular momentum $s$ states. This allows us to expand the electronic wave function in the single excitation basis $\left\{\left|\pi_{k}\right\rangle\right\}$, where $\left|\pi_{k}\right\rangle=|s \ldots p \ldots s\rangle$ denotes a state with the $k$ th atom in the $p$ state $[9,10]$.

\section{A. Rydberg-Rydberg interaction and the electronic Hamiltonian}

Interaction potentials between Rydberg atoms can be determined by diagonalizing a dimer Hamiltonian in a restricted electronic state space, using the dipole-dipole approximation [34]. Here we capture the essential features of these potentials into an effective model, including van-der-Waals (vdW) interactions between two atoms in the same state ( $s s$ or $p p$ ), and resonant dipole-dipole interactions between two atoms in different states $(s p)$, leading to the electronic Hamiltonian,

$$
\hat{H}^{\mathrm{el}}(\mathbf{R})=\hat{H}_{\mathrm{dd}}(\mathbf{R})+\hat{H}_{\mathrm{vdw}}(\mathbf{R}),
$$

with resonant dipole-dipole interaction Hamiltonian,

$$
\hat{H}_{\mathrm{dd}}(\mathbf{R})=-\mu^{2} \sum_{\substack{m, n=1 ; \\ m \neq n}}^{N} R_{m n}^{-3}\left|\pi_{m}\right\rangle\left\langle\pi_{n}\right|,
$$

and nonresonant van-der-Waals interaction,

$$
\hat{H}_{\mathrm{vdw}}(\mathbf{R})=-\mathbb{1} \sum_{\substack{m, n=1 ; \\ m \neq n}}^{N} \frac{C_{6}}{2 R_{m n}^{6}},
$$

where $\mathbb{1}$ is the unit operator in the electronic space and $R_{m n}=\left|\mathbf{R}_{m}-\mathbf{R}_{n}\right|$ the distance between atoms $m$ and $n$. The interactions (2a) are isotropic, determined by the scaled radial matrix element $\mu=d_{v, 1 ; v, 0} / \sqrt{6}$. In Sec. $\mathrm{V}$ and Appendix A we discuss how this simplification can be realized using a magnetic field and isolating specific azimuthal angular momentum states. Using just one coefficient $C_{6}<0$ in (2b) ensures repulsive behavior at very short distances for all electronic states, assuming identical vdW interactions between Rydberg atoms in $s$ and $p$ states for simplicity. Their difference in reality can give rise to interesting effects at shorter distances [13] that are, however, not relevant in our context.

Diagonalizing the electronic Hamiltonian for fixed nuclei,

$$
\hat{H}^{\mathrm{el}}(\mathbf{R})\left|\varphi_{k}(\mathbf{R})\right\rangle=U_{k}(\mathbf{R})\left|\varphi_{k}(\mathbf{R})\right\rangle,
$$

gives the eigenstates $\left|\varphi_{k}(\mathbf{R})\right\rangle$ called Frenkel excitons [28] and the eigenenergies $U_{k}(\mathbf{R})$ which form Born-Oppenheimer surfaces (BO surfaces).

\section{B. Initial state}

The initial state of the system can be written as a direct product,

$$
\left|\Psi^{\mathrm{ini}}\right\rangle=\left|\psi_{\mathrm{el}}^{\mathrm{ini}}\right\rangle \otimes\left|\chi_{\text {nuc }}^{\mathrm{ini}}\right\rangle
$$

where $\left|\psi_{\mathrm{el}}^{\mathrm{ini}}\right\rangle$ is the initial electronic state and $\left|\chi_{\text {nuc }}^{\text {ini }}\right\rangle$ the initial spatial position state. We choose $\left|\chi_{\text {nuc }}^{\text {ini }}\right\rangle$ such that the initial position space representation for $N$ atoms,

$$
\begin{aligned}
\chi_{\text {nuc }}^{\text {ini }}(\mathbf{R}) & =\left\langle\mathbf{R} \mid \chi_{\text {nuc }}^{\text {ini }}\right\rangle, \\
& =\left(2 \pi \sigma^{2}\right)^{-\frac{N}{4}} \prod_{n}^{N} \exp \left(-\frac{\left|\mathbf{R}_{n}-\mathbf{R}_{n}^{(0)}\right|^{2}}{4 \sigma^{2}}\right),
\end{aligned}
$$

is a product of Gaussians, centered on the atoms' initial positions $\mathbf{R}_{n}^{(0)}$. We enforce $\mathbf{R}_{n}=x_{n} \mathbf{e}_{x}$ for atoms on the horizonal chain $\left(n \leqslant N_{x}\right)$ and $\mathbf{R}_{n}=\Delta x_{\text {offset }} \mathbf{e}_{x}+y_{n} \mathbf{e}_{y}$ for atoms on the vertical chain $\left(n>N_{x}\right)$, where $\mathbf{e}_{x, y}$ are unit vectors in the $x, y$ directions and $\Delta x_{\text {offset }}$ is the $x$ position of the vertical chain. This makes sure that the atoms have no initial position uncertainty in directions orthogonal to their chain. The longitudinal width $\sigma$ models an experimental preparation in the lowest oscillator state of a harmonic trap. 


\section{Propagation scheme for atomic motion}

For larger numbers of atoms, solving the time-dependent Schrödinger equation of the full Hamiltonian,

$$
\hat{H}(\mathbf{R})=-\frac{\nabla_{\mathbf{R}}^{2}}{2 M}+\hat{H}^{\mathrm{el}}(\mathbf{R}),
$$

for the motion in $\mathbf{R}$ is not feasible in a reasonable time. Instead, we take the Wigner transform of the atomic Gaussian wave function (5a) as a weighting distribution for initial conditions $\gamma_{i}=\left\{\mathbf{P}_{i}, \mathbf{R}_{i}\right\}$ to propagate classical trajectories fulfilling Newton's equation,

$$
M \ddot{\mathbf{R}}=-\nabla_{\mathbf{R}} U_{s}(\mathbf{R}) .
$$

The mechanical force exerted on the atoms is derived from a single Born-Oppenheimer surface $U_{s}$ of the electronic Hamiltonian. However, we do allow transitions to other adiabatic surfaces $U_{k}$ during the time evolution according to Tully's fewest switching algorithm [35-37]. These jumps are random but occur with the probability for a transition between two surfaces $n$ and $m$, which is proportional to the nonadiabatic coupling vector,

$$
\mathbf{d}_{m n}(\mathbf{R})=\left\langle\varphi_{m}(\mathbf{R})\left|\nabla_{\mathbf{R}}\right| \varphi_{n}(\mathbf{R})\right\rangle .
$$

Simultaneously, we propagate the electronic wave function of the Rydberg aggregate $|\Phi(\mathbf{R}(t))\rangle=\sum_{n} c_{n}(t)\left|\pi_{n}\right\rangle$, expressed in the atomic (diabatic) basis $\left\{\left|\pi_{n}\right\rangle\right\}$, by solving the matrix equations for the coefficients $c_{n}(t)$,

$$
\mathrm{i} \hbar \dot{c}_{k}(t)=\sum_{\ell} \hat{H}_{k \ell}^{\mathrm{el}}(t) c_{\ell}(t),
$$

where

$$
\begin{aligned}
\hat{H}_{k \ell}^{\mathrm{el}}(t) & \equiv\left\langle\pi_{k}\left|H^{\mathrm{el}}(\mathbf{R}(t))\right| \pi_{\ell}\right\rangle \\
& =-\frac{\mu^{2}}{R_{k \ell}^{3}(t)}-\left(\sum_{\substack{m, n=1 ; \\
m \neq n}}^{N} \frac{C_{6}}{2 R_{m n}^{6}(t)}\right) \delta_{k \ell} .
\end{aligned}
$$

In addition to coefficients in the diabatic basis, we will also refer to those in the adiabatic basis defined by $|\Phi(\mathbf{R}(t))\rangle=$ $\sum_{n} \tilde{c}_{n}(t)\left|\varphi_{n}(\mathbf{R}(t))\right\rangle$. Each trajectory is a self-consistent solution of the coupled atomic and electronic equations of motion (7) and (9), taking into account in addition the random jump sequence $s$ among the BO surfaces discussed above. With a recorded jump-sequence $s_{i}(t)$, each trajectory $i$ represents a realization. More precisely, the propagation sequence for a single trajectory with initial conditions $\gamma_{i}$ at time $t=0$ starting on the BO surface $U_{s_{i}(0)}$ consists of the following steps:

(1) The electronic Hamiltonian is diagonalized and at any given time a single eigenenergy $U_{s(t)}(\mathbf{R}(t))$ is the potential which exerts force on the atoms.

(2) The atomic positions are propagated with (7) and the electronic states with (9) for one time step $\Delta t$.

(3) We determine whether the surface index $s_{i}$ undergoes a stochastic jump after this time step to $s_{i}(t+\Delta t) \neq s_{i}(t)$ (see [9] for the precise prescription).

(4) The new positions $\mathbf{R}(t+\Delta t)$ lead to new eigenstates and eigenenergies. Hence, we repeat the procedure from point II C with updated time variable $t \leftarrow t+\Delta t$.
We use a single realization $s_{i}$ for each initial condition $\gamma_{i}$, which is in practice sufficient to converge the stochastic character of the jumps between surfaces if a large number of trajectories is propagated whose initial conditions randomly sample the initial Wigner distribution. Results obtained in this way agree very well with full quantum results for three atoms where quantum calculations can be done [10,11,14,31]. Since Tully's algorithm is expected to be an even better approximation for a larger number of dimension we confidently have adapted it to our present problem as described above.

\section{NONADIABATIC DYNAMICS}

Equipped with the tools to describe dynamics where the aggregate can make transitions between different adiabatic surfaces or excitons, we would like to control these transitions in order to steer exciton transfer in a complex landscape of potential surfaces. It is well known that conical intersectionswhere adiabatic surfaces touch each other-lead to enhanced nonadiabatic transfer from one surface to another. Previously, we investigated transport of excitation and entanglement in linear flexible Rydberg chains without nonadiabatic transfer $[10,11]$, and the opposite, nonadiabatic processes near conical intersections without transport in ring configurations [12].

A system exhibiting both features, transport and induced nonadiabatic transfer, is a T-shape configuration of two onedimensional chains of atoms. We will present their dynamics with three and four atoms, respectively, on the two chains, using Li atoms with a mass of $M=11000 \mathrm{au}$. First, however, we investigate the simplest possible realization, a double dimer with two atoms on each of two orthogonal lines as sketched in Fig. 1.

\section{A. Two perpendicular dimers}

We use Rydberg states in principal quantum number $v=$ 44 , leading to a transition dipole moment of $\mu=1000 \mathrm{au}$. For simplicity we set $C_{6}=0$ in this section. The parameters of the initial atomic configuration were set to $a_{1}=2.16 \mu \mathrm{m}$, $a_{2}=5.25 \mu \mathrm{m}$, and $d=8.5 \mu \mathrm{m}$. The $x$ position of the vertical chain is fixed to $\Delta x_{\text {offset }}=a_{1}+d$. To sample the initial nuclear (Wigner) distribution with Gaussian width of $\sigma=0.5 \mu \mathrm{m}$ about each atomic position (5a), we have propagated $10^{5}$ trajectories.

A single $p$ excitation in the system shared between atoms 0 and 1 leads to their mutual repulsion as indicated by the blue arrows in Fig. 1(a) and enables atom 1 to move towards the vertical chain. On this way the three atoms 1-3 form a triangular subunit corresponding to the ring trimer studied in [12]. The CI of the trimer is realized for $p=1, d=\sqrt{3} / 2 a_{2}$ in Fig. 1(a), where the three atoms form an equilateral triangle. To illustrate the CI, we show in Fig. 1(b) the two intersecting energy surfaces as a function of two selected atomic position variables. The upper surface, shaded in red, will be hereafter referred to as the repulsive surface $U_{\text {rep }}$ (with exciton state $\left.\left|\varphi_{\text {rep }}\right\rangle\right)$, as it always entails repulsive interactions of nearby atoms. The lower surface at the intersection, shaded in green, will be referred to as the adjacent surface $U_{\text {adj }}$ (with exciton state $\left|\varphi_{\text {adj }}\right\rangle$ [38]). Further surfaces are not shown and play no significant role. 
We will now systematically construct and interpret the atomic motion triggered by the initial excitation, first by analyzing typical trajectories and their energy spectra, then by constructing the atomic densities on the repulsive and adjacent adiabatic surface, which finally will enable us to understand the full evolution of the atomic density. We consider its evolution in time, spatially resolved, in terms of population of adiabatic surfaces and regarding the purity of the state.

\section{Evolution and energy spectra of typical trajectories}

Since we neglect all uncertainties transverse to each of the trapping beams [see (5a)], atoms zero and one have well-defined $y$ coordinates. However, the atoms on the vertical line have a distribution in $y$ about their central position such that different trajectories have different asymmetry parameters $p$. We distinguish the "symmetric" part of the nuclear wave packet with $p \approx 1$ [a typical trajectory is shown in Fig. 2(e)] from the "asymmetric" part which realizes other $p$ values (two trajectories that are nearly related through vertical mirroring
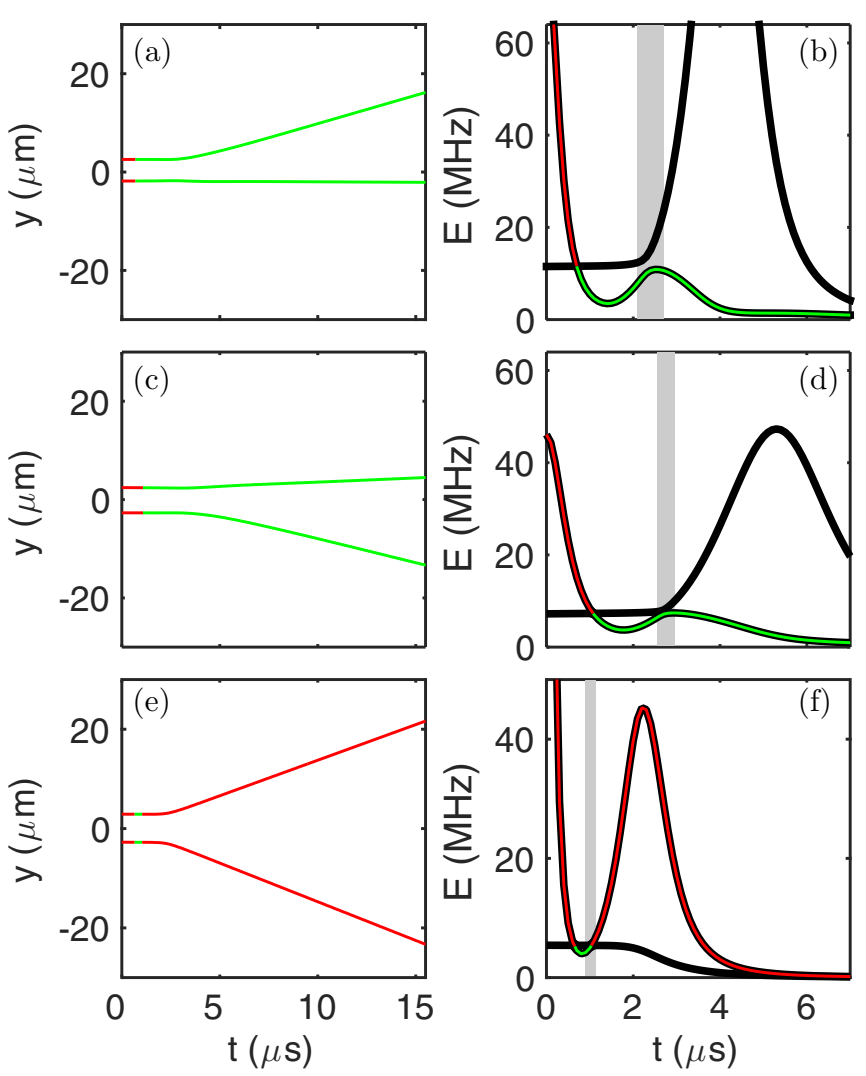

FIG. 2. Selected single trajectories $\mathbf{R}(t)$ of atoms 2 and 3 in (a), (c), and (e) with corresponding time-resolved energy spectra (black lines) and potential energy $U_{s(t)}(\mathbf{R}(t))$ (colored line) in (b), (d), and (f). For both, trajectories and potential energy, evolution on the repulsive surface is marked with red lines and evolution on the adjacent surface with green lines. (a) and (c) Two characteristic trajectories that stay on the adjacent surface. The two trajectories are almost mirror symmetric around the $x$ axis. (e) A characteristic trajectory jumping back to the repulsive surface after passing the vicinity of the CI. The gray area marks the vicinity of the CI between the adjacent and the repulsive surfaces, ruling the dynamics. The earlier crossings are less relevant for our dynamics here; see [39]. are shown in Figs. 2(a)-2(c). One can see that initially, symmetric and asymmetric trajectories evolve very similarly. In particular, both of them jump from the repulsive surface (red) to the one we have called the adjacent surface of the trimer (green) a bit before one $\mu s$ into the dynamics; see the energy spectra Figs. 2(b), 2(d), and 2(f). This point in parameter space represents a further conical intersection with only trivial consequences for our dynamics here; see [39]. During this initial time, atoms 0 and 1 simply repel (not shown), until atom 1 sets 2 and 3 into motion around $2 \mu \mathrm{s}$. Shortly afterwards (during the gray shaded time interval) the dynamics of the trajectories is ruled by the essential conical intersection of the trimer. Here, the symmetric part, coming near the degenerate point of the CI, is more likely to make a transition to the repulsive surface than the asymmetric part, which stays dominantly on the adjacent surface and passes the CI at a greater distance; see Fig. 1(b). This is reflected in the energy spectrum of the trajectories by a very narrow avoided crossing (it appears actually as a crossing within the resolution of Fig. 1(f) for the symmetric trajectory in the gray shaded area. Consequently, the symmetric trajectory follows a diabatic path, jumping from the adjacent surface to the repulsive one, while the two asymmetric trajectories that miss the CI move adiabatically in their energy landscape Figs. 2(b) and 2(d), with a relevant avoided crossing in the gray shaded area that is wider allowing them to stay on the adjacent surface. A final transition to the repulsive surface leads to a significant separation of atoms 2 and 3 in the course of time, while the asymmetric trajectories that stay on the adjacent surface contain one atom remaining almost at rest, while the other one, initially at larger distance to the horizontal chain experiences the stronger force [Fig. 1(c)] and moves away [40].

Exciton spectra such as shown in Fig. 2 should be observable with microwave spectroscopy of Rydberg aggregates, such as in $[19,41]$.

\section{Atomic densities on the adiabatic surfaces forming the conical intersection}

To evolve the Rydberg aggregate from its initial (Wigner) distribution, we have calculated $i=1, \ldots, N_{\text {traj }}=10^{5}$ trajectories, the surface index $s_{i}(t)$ of which at each instant of time allows us to show the atomic density [42] on the adjacent and repulsive surface separately as done in Fig. 3 for atoms 2 and 3. One directly recognizes that the wave packet enters the CI on the adjacent surface [no density in Fig. 3(b) for short times] and that through the $\mathrm{CI}$ roughly half of the density is transferred to the repulsive surface where the two repelling branches show a wide distribution. On the adjacent surface, on the other hand, the fourfold branching is due to the asymmetric character of its individual trajectories as illustrated in Figs. 2(a) and 2(c).

\section{Time-dependent observables of the double dimer}

We finally present the full time evolution of the double dimer in Fig. 4, where we clearly recognize in (a) the initial repulsion of atoms 0 and 1 of the horizontal chain. Furthermore, we can appreciate and understand the complicated fanning out of density in the vertical chain after the CI as a superposition of the wide two-branch distribution on the repulsive surface and the narrower four finger double fork on the adjacent surface 

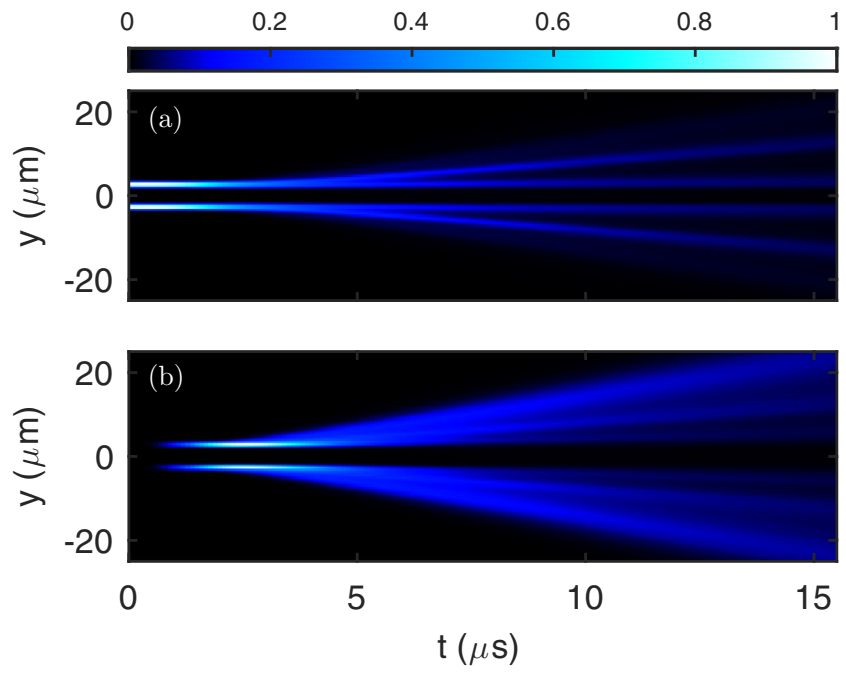

FIG. 3. Atomic densities of atoms 2 and 3 on the adjacent surface (a), and on the repulsive surface (b). Atomic positions were binned into one or the other according to the trajectory surface index $s$. The maximum value of the data over both (a) and (b) is set to unity with a common normalization.
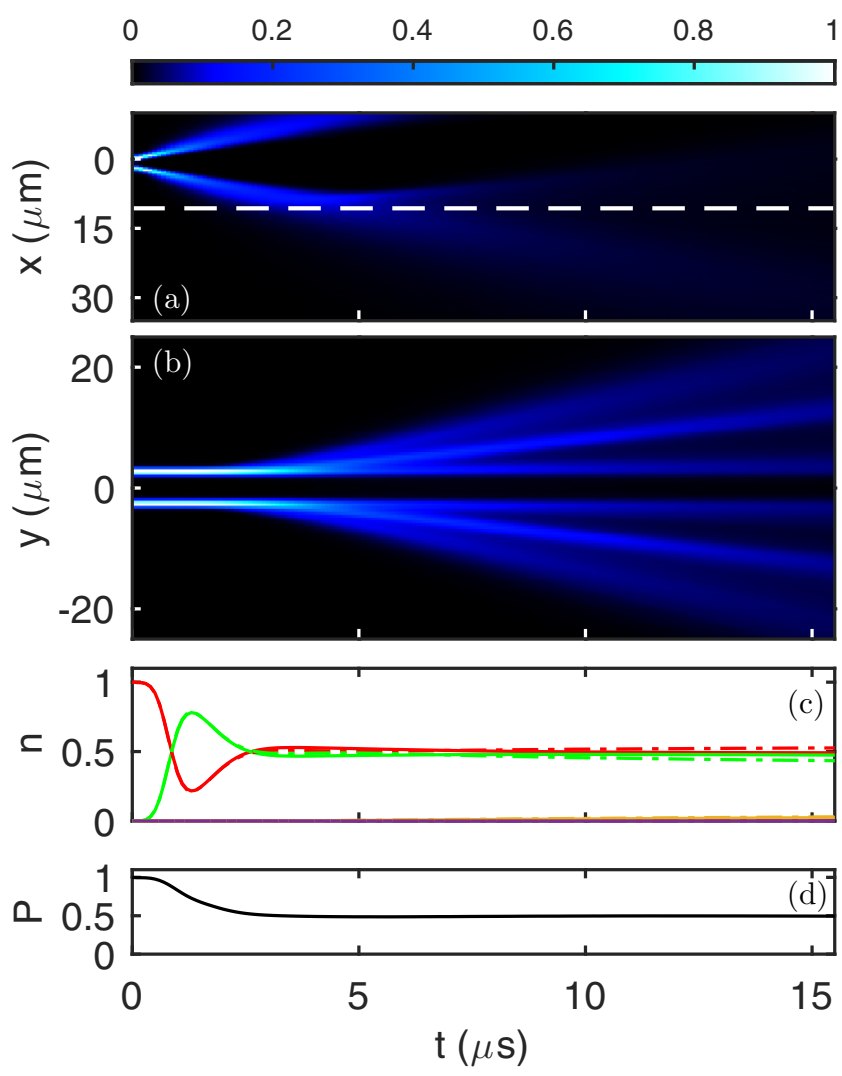

FIG. 4. Dynamics of atomic motion and electronic populations; total atomic density of atoms 0 and 1 in (a) and atoms 2 and 3 in (b). (c) Adiabatic populations, $\overline{\left|\tilde{c}_{n}\right|^{2}}$, (solid lines) and trajectory fractions $f_{n}=\sum_{i}^{N_{\text {traj }}} \delta_{n, s_{i}(t)} / N_{\text {traj }}$ (dashed dotted lines) on the repulsive surface (red) and adjacent surface (green). Here $\cdots$ denotes a trajectory average. (d) Purity $P=\operatorname{Tr}\left[\hat{\sigma}^{2}\right]$ of the reduced electronic density matrix $[10,43]$. All results are averaged over $10^{5}$ realizations. The maximum value of the data in (a) and (b) is individually set to one.
[Fig. 4(b)]. The population on both surfaces as a function of time in Fig. 4(c) confirms that the wave packet starts out on the repulsive surface (red) and continues after the first crossing on the adjacent surface (green), while it is split at the conical intersection to finally populate both surfaces roughly equally. As a consequence, the purity [43] of the wave packet decreases from unity to one-half in Fig. 4(d).

\section{B. The seven atom T-shape system}

The double dimer was a necessary prerequisite to create a coherent exciton pulse along a path defined by atoms positioned in space: We now know that the atoms on the vertical chain will not attract each other after the atomic wave packet has passed the CI crossing, which is the first condition to continue excitation transport from the horizontal onto the vertical chain. The transport from its initiation, by creating a repulsive exciton localized on the first two atoms, to the $\mathrm{CI}$ on the horizontal chain follows the dynamics we have already established with "Newton's cradle" in Ref. [10]. However, for more atoms in the vertical chain, the CI may induce features which qualitatively go beyond what we have seen in the double dimer. Hence we extend our system to three atoms in the horizontal and four atoms in the vertical chain, keeping the configuration of two orthogonal lines as shown in Fig. 5. The resulting setup corresponds to two linear atomic "Newton's Cradle" configurations as in Ref. [10] that intersect at a right angle, such that transport involves a CI.

\section{Atomic configuration}

We place the atoms initially as shown in Fig. 5 and prepare the system in the repulsive exciton localized on atoms 0 and 1 . As before, this leads to a repulsive motion, such that atom 1 kicks atom 2, which eventually transfers its energy and electronic excitation to atom 2 as familiar from Newton's cradle. Once atom 2 reaches $x_{\mathrm{CI}}$ the system can again be understood by considering the atomic trimer now formed by atoms 2, 4, and 5 .

\section{Fully coherent exciton transport to the vertical chain}

The simplest case essentially involves only a single BO surface. For sufficient, negative $\Delta y$, atom 2 misses the $\mathrm{CI}$ and traverses the $y$ axis much closer to atom 5 than to atom 4 . In this case Newton's cradle like binary exciton transfer from atom 0 to 1 over 1 to 2 on the horizontal chain will carry over to upward transport on the vertical chain via transfer from atom 2 to 5 and ultimately 5 to 6 , realizing fully adiabatic dynamics with respect to the energy surfaces. Analogously one gets for positive $\Delta y$ ultimate exciton transfer to atoms 4 and 3 . Hence changes in $\Delta y$ switch between downwards or upwards exciton transport, as discussed further in Sec. IV.

Less clear is what happens with the excitation and entanglement transport if atom 2 approaches the vertical chain with $\Delta y \approx 0$. As we know from the double dimer, motion of atoms 4 and 5 is then affected by the CI through dynamics on its constituting energy surfaces, the adjacent and the repulsive one. To elucidate what this means for the ensuing excitation transport on the vertical chain we present calculations with Rydberg excitation $v=80$, which gives a transition dipole 


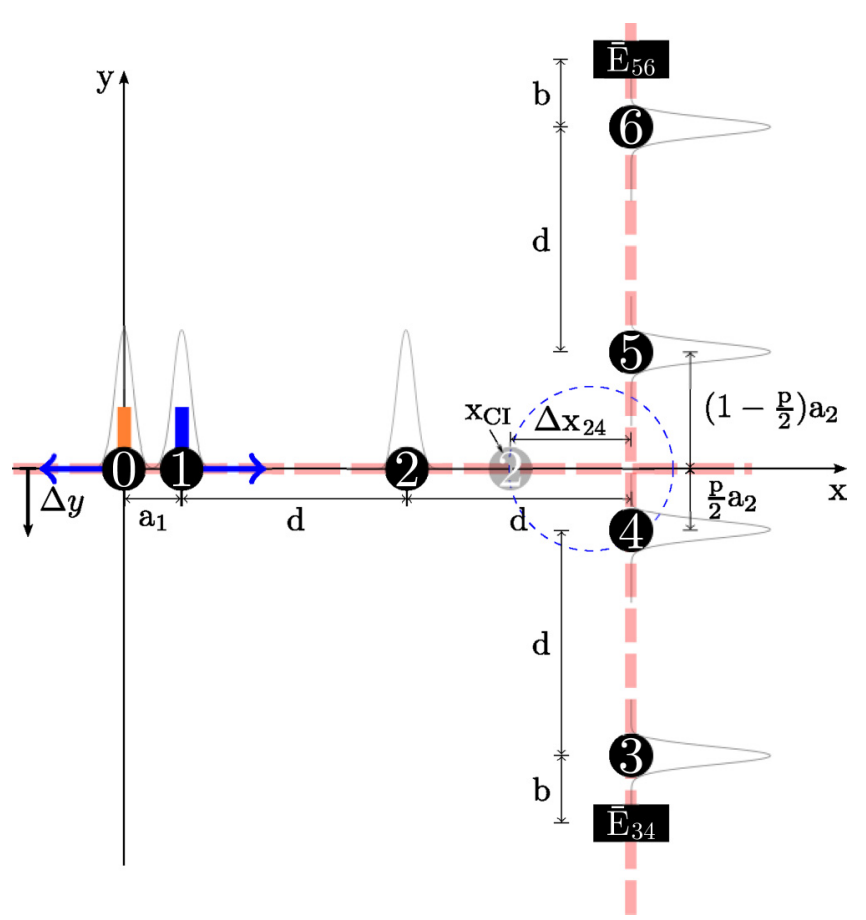

FIG. 5. Sketch of a seven-atom system combining features of adiabatic entanglement transport and CI dynamics. Three atoms are placed on the horizontal line and four on the vertical one. Atoms 0 and 1 are prepared in a repulsive exciton at the initial time $t_{0}$, resulting in the excitation distribution shown by orange and blue bars, as described in the caption of Fig. 1. Atom 2 comes close to a CI configuration, when it is at position $x_{\mathrm{CI}}$. Around that moment, the excitation almost exclusively resides on atoms 2,4 , and 5 , since all other atoms are far away from this trimer. Atoms 4 and 5 are accelerated at this time on the two different surfaces, already described in the fouratom section. We analyze whether or not the atom pair $(3,4)[(5,6)]$ finally contains an exciton-motion pulse propagating in the negative (positive) $y$ direction. This is quantified by the binary entanglement [10] $E_{34}\left[E_{56}\right]$ at the moment that atom 3 [5] reaches the location indicated by a black box, termed "entanglement readout." We finally use the indicated displacement $\Delta y$ of the horizontal chain to steer entanglement transport upwards or downwards.

moment of $\mu=3371$ au of the lithium atoms. The parameters of the perpendicular chains are $a_{1}=6 \mu \mathrm{m}, a_{2}=9.5 \mu \mathrm{m}$, $d=22 \mu \mathrm{m}, \Delta \mathrm{x}_{\text {offset }}=a 1+2 d$ and we use the same initial Gaussian distributions (5a) for the atomic positions.

\section{Simultaneous dynamics on several surfaces}

We first discuss the case $\Delta y=0 \mu \mathrm{m}$ implying that the incoming atomic wave packet directly hits the CI.

The total atomic density on the horizontal chain Fig. 6(a) clearly shows the repulsive behavior of the atoms transferring the initial momentum and excitation shared by atoms 0 and 1 from atom 1 to atom 2. Once atom 2 approaches the vertical chain it enters the region of the CI which leads, as for the double dimer, to a distribution of the atomic density on the vertical chain over the repulsive and adjacent surface [Fig. 6(b)]. The exciton spectra of selected single trajectories on these surfaces shown in Fig. 7 behave quite similarly as for the double dimer. In the beginning on the horizontal
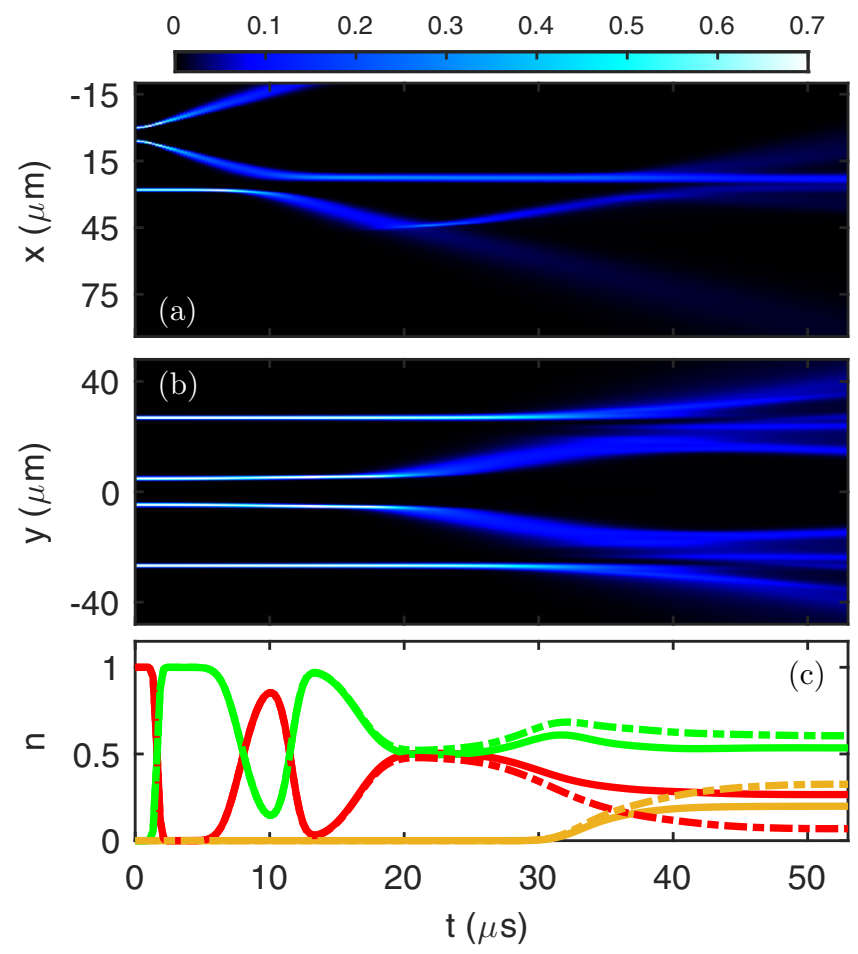

FIG. 6. Dynamics of atomic motion and populations. (a) Total atomic density of atom 0 to 2. (b) Total atomic density of atom 3 to 6. (c) Adiabatic populations (solid lines) and fractions (dashed dotted lines) of repulsive surface (red), second most energetic (adjacent) surface (green), and third most energetic surface (yellow). All data are averaged over 1 million realizations. The maximum value of the data in (a) and (b) is individually set to one. To highlight details at lower densities, all values between 0.7 and 1 are represented with white.

chain the trajectories switch diabatically a couple of times between the adjacent and repulsive surface before their paths finally become qualitatively different close to the CI (gray shaded region) when the two asymmetric trajectories shown in Figs. 7(a)-7(d) stay on the adjacent surface, which then rules their subsequent dynamics on the vertical chain, while the more symmetric trajectory of Figs. 7(e) and 7(f) jumps to the repulsive surface. The main difference compared to Fig. 4 that one recognizes is the transfer of momentum to the two new outer atoms 3 and 6 on the vertical chain.

\section{Inversion of excitation transport direction on the adjacent surface}

Based on the understanding gained in Sec. III A 1, we can now understand an essential qualitative difference between transport dynamics on the repulsive and adjacent surfaces. To this end, we visualize in Fig. 8 the allocation of excitation transport onto the different atoms of the vertical chain, for two cases that differ only in the parameter $a_{2}$. While in both cases, atom 2 passes most closely to atom 4 , the repulsive surface realizes the intuitively expected transport continuing with atom 4, while the adjacent surface instead leads to transport continued with atom 5 in the opposite direction. As explained in Sec. III A 1, this can be directly traced back to differences 

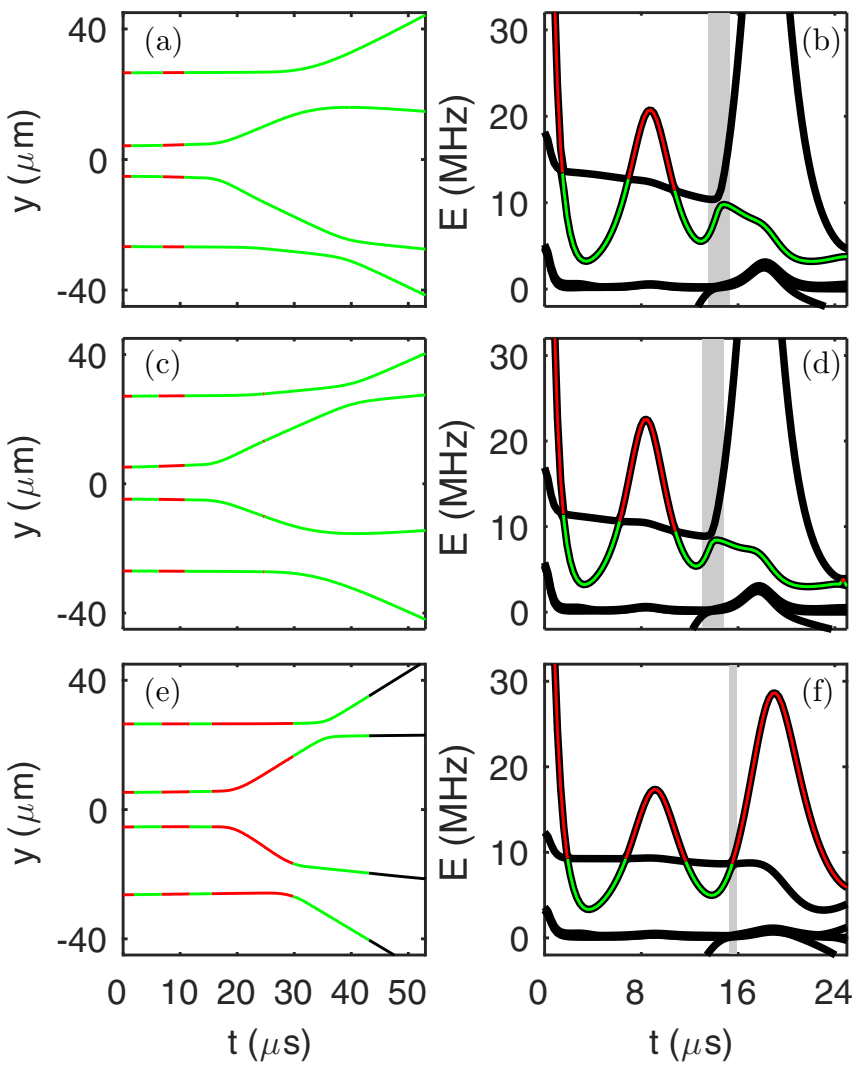

FIG. 7. Atomic positions from single trajectories for atoms 3-6 (a), (c), and (e) with corresponding eigenenergies in (b), (d), and (f) for $\Delta y=0 \mu \mathrm{m}, a_{2}=9.5 \mu \mathrm{m}$. (a) and (c) Two different trajectories ending up on the adjacent surface. (e) A trajectory returning to the repulsive surface after being in the vicinity of the CI. (b), (d), and (f) Time-resolved energy spectra (black lines) and potential energy (colored line), for position trajectories on the left. For both, positions and potential energy, evolution on the repulsive surface is marked with red and evolution on the adjacent surface with green. The trajectories shown in (e) evolve in the end on a third surface, marked with black lines. The gray area marks the CI and its vicinity. For a discussion of the earlier curve crossings see [39].

between the repulsive and adjacent exciton states near the essential conical intersection.

\section{EXCITON SWITCH}

Depending on how the passage through or near the CI distributes population onto the repulsive or adjacent energy surface, either of the transport mechanisms discussed above can be made dominant. This population distribution depends on the width and position of the multidimensional atomic wave packet describing all coordinates $\mathbf{R}$, as well as the effective size of the CI which is essentially controlled by the corresponding multidimensional velocity $\dot{\mathbf{R}}$ [12]. All these features can be varied as a function of our configuration parameters $a_{1}, a_{2}, d$, and $\Delta y$.

Extending the work of [31] we show a parameter space survey of the ensuing "exciton switch" in Fig. 9. To characterize the exciton transport in a given configuration, we use the binary entanglement of the final atoms on the vertical chain, at

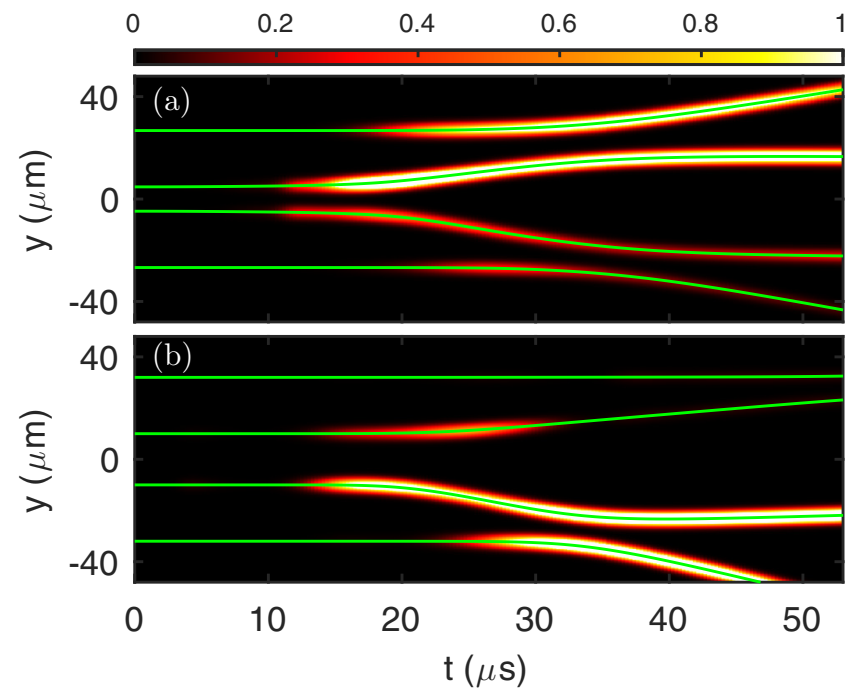

FIG. 8. Qualitatively different dynamics on the repulsive and adjacent surface. Mean atomic positions (green lines), with $p$ excitation probability of the combined repulsive and adjacent surfaces (black-red-yellow-white shades) on each atom of the vertical chain, $\Delta y=1.5 \mu \mathrm{m}$. (a) Case that mostly remains on the adjacent surface, with $a_{2}=9.5 \mu \mathrm{m}$. (b) Dominantly repulsive case with $a_{2}=20 \mu \mathrm{m}$. The excitation probability for atom $n$ is represented by Gaussians of a selected fixed width, normalized to $\sum_{n}\left|\left\langle\pi_{n} \mid \varphi_{\mathrm{n}}(\mathbf{R})\right\rangle\right|^{2}$ (where $\sum_{n}$ runs over the repulsive and adjacent surfaces) and centered on the mean position of atom $n$.
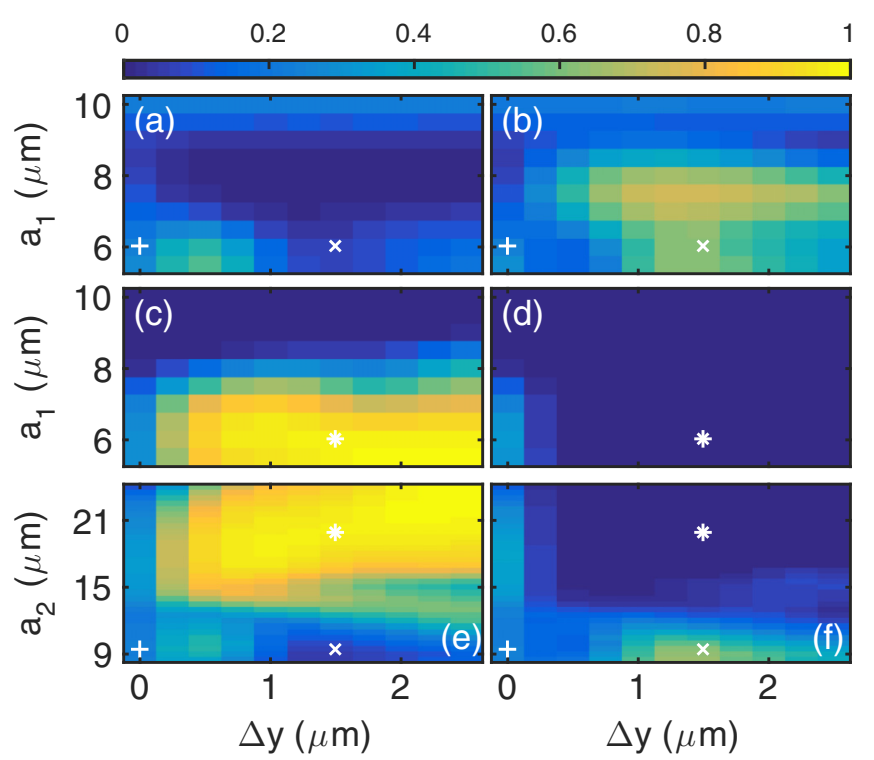

FIG. 9. Response of the exciton switch to control parameters. We show the bipartite entanglement transported downwards in $y, \bar{E}_{34}$ (left column) and upwards, $\bar{E}_{56}$ (right column). Parameters are $v=80$ and $d=22 \mu \mathrm{m}$. Both entanglement readouts (see Fig. 5) are placed in a distance of $b=0.3 d$ from atoms 3 and 6 , respectively, on the vertical chain. (a)-(d) Entanglement as a function of $a_{1}$ and $\Delta y$, for $a_{2}=9.5 \mu \mathrm{m}$ in (a) and (b) and $a_{2}=20 \mu \mathrm{m}$ in (c) and (d). (e) and (f) Entanglement as a function of $a_{2}$ and $\Delta y$, for fixed $a_{1}=6 \mu \mathrm{m}$. The markers $(*, \times,+)$ highlight extreme cases already presented in [31]: $(*)$ high entanglement transport to atoms $(3,4),(\times)$ high entanglement transport to atoms $(5,6),(+)$ equal entanglement transport towards atoms $(3,4)$ and atoms $(5,6)$. 
the moment when the first of them reaches its "entanglement readout" location, described in Fig. 5. For a definition of the binary electronic entanglement $\bar{E}_{i j}$ of two neighboring atoms $i, j$; see [10]. A large value of $\bar{E}_{34}\left[\bar{E}_{56}\right]$ corresponds to a coherent exciton-motion pulse traveling in the downward (upward) direction.

For various combinations of $a_{1}, a_{2}$, and $\Delta y$, we plot $\bar{E}_{34}$ and $\bar{E}_{56}$ in Fig. 9, determined numerically from simulations as in Fig. 6 with a reduced number of trajectories. The three cases selected in [31] are shown as symbols $(*, \times$ and +$)$. To understand the inversion of the entanglement transport direction for varying $a_{2}$ at fixed $\Delta y$, please refer to our discussion in Sec. III B 4.

\section{ISOTROPIC VERSUS ANISOTROPIC DIPOLE-DIPOLE MODEL}

The model of interactions (1) employed so far was isotropic, neglecting the angular dependence of dipole-dipole interactions [34,44], and the presence of several azimuthal sublevels of the Rydberg $|p\rangle$ state. In general we have to expand our basis to $\left|\pi_{n}, m_{l}\right\rangle=\left|s \ldots\left(p, m_{l}\right) \ldots s\right\rangle$, where $m_{l}$ is the magnetic quantum number of atom $n$ in the $|p\rangle$ state. For a one-dimensional atomic chain, the model (1) with basis $\left|\pi_{n}\right\rangle$ can be recovered by restricting the initial state to $m_{l}=0$, when the quantization axis is parallel to the atomic chain [11]. Dipole-dipole interactions then do not populate any other azimuthal states.

For the phenomena discussed in this article, we require $\hat{V}_{\mathrm{dd}}(\mathbf{R})<0$ at all $\mathbf{R}$, for the system to display the conical intersection between the repulsive and adjacent surface. Since additionally atomic distances here span two spatial dimensions, the procedure to eliminate the azimuthal degree of freedom described above is no longer possible. To ensure the correct sign of interactions in this case, we concentrate on the azimuthal sublevels $m_{l}=1$ with quantization axis perpendicular to the $x-y$ plane. To prevent undesired coupling to the $m_{l}=-1$ states, we additionally impose a uniform external magnetic field $\mathbf{B}=B_{z} \hat{\mathbf{z}}$, where $\hat{z}$ is a unit vector in the $z$ direction. This creates an energy offset $\Delta E=m_{l} \mu_{B} B_{z}$ for all electronic states, effectively decoupling those with $m_{l}=-1$ from $m_{l}=1$. We discuss formally in Appendix A how this results in our isotropic model (1) in the limit of an infinitely strong magnetic field, and how corrections to this model for realistic field strengths can be calculated. These corrections leave the qualitative results unchanged as we will show.

To this end we now repeat the simulations of Fig. 4 using a model that allows all magnetic states for all four atoms in the presence of an external magnetic field, described in Appendix A and further in [45]. The motion of the atoms is still constrained onto two orthogonal lines, as in the rest of the article. We demonstrate in Fig. 10 that qualitatively the same main features are found as for the isotropic model. However, for the greatest resemblance the parameters of both models have to be chosen slightly different due to the quantitative difference of potentials, which affect most importantly the initial acceleration of atoms 0 and 1 , in turn controlling the relative population of the two energy surfaces after CI crossing, seen in panel (e). For Fig. 10 we have adjusted $a_{1}$ in both models separately to achieve a rough 50-50 splitting
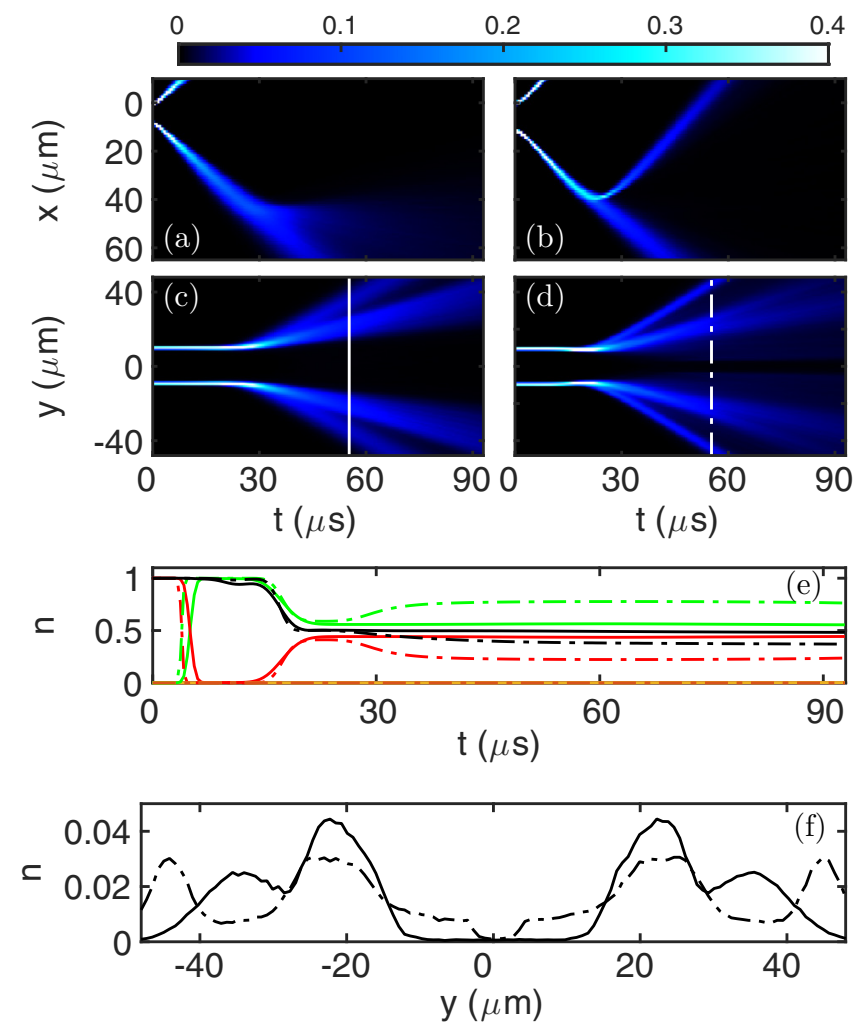

FIG. 10. For the case of Fig. 4, we compare our model of Sec. II with one additionally allowing different azimuthal sublevels $m_{l}$, anisotropic interactions and a magnetic field; see Appendix A. In both models we used the parameters $v=80, a_{2}=19 \mu \mathrm{m}$ and $d=40 \mu \mathrm{m}$. To obtain qualitatively the same dynamics in both, $a_{1}$ is adjusted individually, compensating quantitative differences in potentials. The anisotropic model uses $a_{1}=8 \mu \mathrm{m}$ and the isotropic one $a_{1}=11.8 \mu \mathrm{m}$. (a) and (c) Atomic density of atom 0 and atom 1 . (b) and (d) Atomic density of atoms 2 and 3, where (a) and (c) are for the anisotropic model and (b) and (d) for the isotropic one. To highlight details at lower densities, all values between 0.4 and 1 are represented with white. (e) Adiabatic populations on the (red line) repulsive and (green line) adjacent energy surface and purity (black lines). We compare the anisotropic (solid line) with the isotropic model (dash-dotted line). (f) Cut through the atomic densities at the time indicated by white lines in (b) and (d) for both models, with line styles as in (e).

on the two surfaces. Both variants then qualitatively agree, in particular regarding clear signatures of multiple populated Born Oppenheimer surfaces in the snapshot shown in panel (f).

\section{CONCLUSIONS}

We have considered assemblies of dipole-dipole interacting Rydberg systems, whose motion and quantum state dynamics is affected by a conical intersection. For atoms free to move on either of two orthogonal chains, we have shown that the CI in the spectrum acts as a selector, through which parts of the wave packet where atoms are spaced symmetrically around one of the chains tend to be transferred to the repulsive surface, while the rest remains on the adjacent surface. As a result of the splitting onto two surfaces, the total atomic density shows 
several characteristic features, clearly signaling the crossing of a conical intersection.

The CI subunit of the two chains, with four atoms in two orthogonal dimers, can be understood as a building block for more complex systems. The block allows branching and switching of dislocation pulses within the system. Since the fraction of total population on either of the surfaces sensitively depends on the motion velocity and the width of the nuclear wave packet, the switching can be tuned or externally controlled. We have shown that this can be achieved through small changes of the confinement geometry, extending the work of [31] by a detailed parameter space survey of the ensuing exciton switch. Possible future extensions could be velocity adjustments of Rydberg atoms via external fields, e.g., [46].

In this article we have employed an isotropic interaction model with just one participating azimuthal quantum number, which results in numerical simplifications and easier interpretation of quantum dynamics. We have demonstrated how this model can experimentally be realized using a magnetic field. However, the essential physics reported, the splitting of a Rydberg atom wave packet onto multiple Born-Oppenheimer surfaces through acceleration by resonant dipole-dipole interactions is a generic feature of higher dimensional Rydberg aggregates. It neither relies on any of the above simplifications (as shown here) nor on the confinement of atomic motion on one-dimensional lines (as we will report in [45]).

Finally, note that similar transport processes on different energy scales could be studied through Rydberg dressed dipole-dipole interactions [47-49], or could rely on Rydberg atoms immersed in host cold atom clouds [14,50] instead of individual atoms.

\section{ACKNOWLEDGMENTS}

We gratefully acknowledge fruitful discussions with Alexander Eisfeld and Sebastian Möbius.

\section{APPENDIX：ISOTROPIC DIPOLE-DIPOLE INTERACTIONS}

As demonstrated in Sec. V, an external magnetic field can be used to reduce the number of electronic angular momentum states participating in the dynamics of Rydberg aggregates and thus render the interactions predominantly isotropic. Here we describe the underlying model and analytically derive an expression for the resulting effective interactions, including anisotropic correction terms. We additionally discuss spin of the Rydberg atoms, and show that the same mechanism that renders interactions isotropic also removes spin as a degree of freedom.

Besides including the azimuthal quantum number in our extended single excitation basis $\left|\pi_{n}, m_{l}\right\rangle$ (see Sec. V), we also have to describe the spin of the atoms, where it suffices to label the $z$ component for each atom $i$ by $m_{s}^{(i)}$. We start by defining the Hamiltonian for the interaction of the magnetic field with $N$ atoms, given by

$$
\hat{H}_{\mathcal{M F}}\left(B_{z}\right)=\mu_{\mathrm{B}} B_{z} \sum_{i=1}^{N} \hat{L}_{z}^{(i)}+2 \hat{S}_{z}^{(i)} .
$$

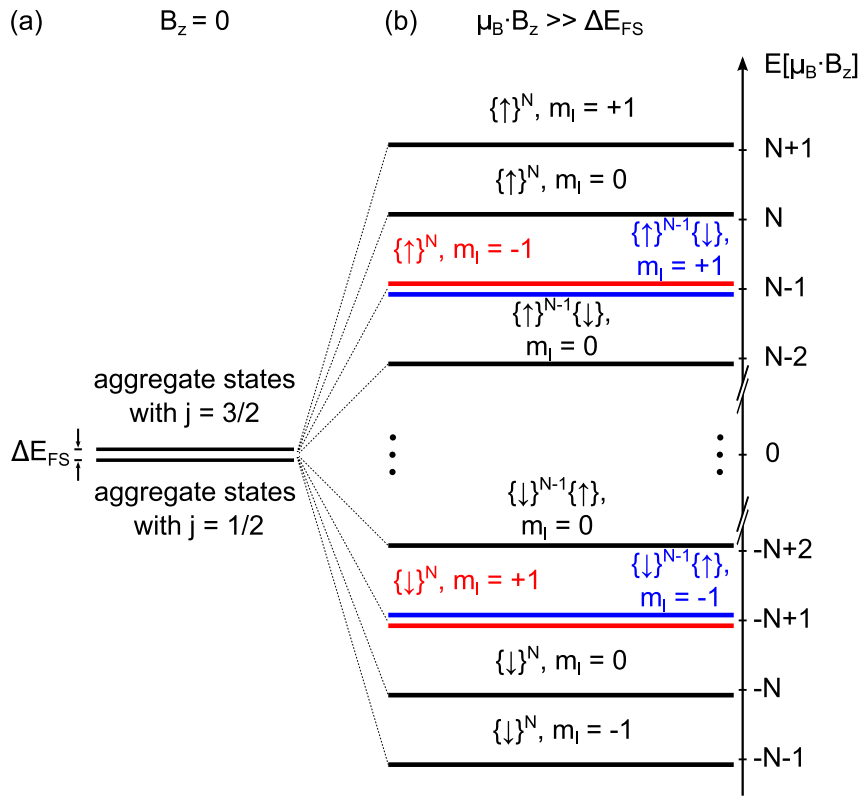

FIG. 11. Sketch of energy splittings for the aggregate states. (a) Without magnetic field, the fine structure separates aggregate states where the $p$ states have total angular momentum $j=1 / 2$ from the ones with $j=3 / 2$. (b) An external magnetic field within the strong field regime, where $\mu_{B} \cdot B_{z} \gg \Delta E_{F S}$, splits the aggregate states into different manifolds with energy gaps $\mu_{B} \cdot B_{z}$ for $\left(\Delta M_{S}, \Delta m_{l}\right)=$ $(0, \pm 1)$ and energy gaps of the order of $\Delta E_{F S}$, for $\left(\Delta M_{S}, \Delta m_{l}\right)=$ $( \pm 1, \mp 2)$ (energy gap between neighboring blue and red lines). The notation $\{\uparrow\}^{N_{\uparrow}}\{\downarrow\}^{N_{\downarrow}}$ is a short form for a spin configuration with $N_{\uparrow}$ spins oriented upwards $\left(m_{s}=1 / 2\right)$ and $N_{\downarrow}$ spins oriented downwards $\left(m_{s}=-1 / 2\right)$.

The magnetic field $B_{z}$ points in the $z$ direction and $\hat{L}_{z}^{(i)}, \hat{S}_{z}^{(i)}$ denote the operators of angular momentum and spin in the $z$ direction for the $i$ th atom, and $\mu_{\mathrm{B}}$ is the Bohr magneton. A spin configuration for the aggregate is uniquely defined by the tuple $\mathbf{M}_{S}:=\left[m_{s}^{(1)} \ldots m_{s}^{(N)}\right]^{\mathrm{T}}$. We denote the corresponding state with $\left|\mathbf{M}_{S}\right\rangle:=\left|m_{s}^{(1)}\right\rangle_{1} \ldots\left|m_{s}^{(N)}\right\rangle_{N}$, which is the product state of all single atom spin states, labeled for the $i$ th atom with $\left|m_{s}^{(i)}\right\rangle_{i}$. Introducing the quantum number for the $z$ component of the aggregate spin,

$$
M_{S}\left(\mathbf{M}_{S}\right):=\sum_{i=1}^{N} m_{s}^{(i)},
$$

which is the sum over all individual spin quantum numbers, the energy shift of the magnetic field is for $\left(M_{S}, m_{l}\right)$ states given by

$$
\Delta E_{\mathcal{M F}}\left(B_{z}, M_{S}, m_{l}\right)=\mu_{\mathrm{B}} B_{z}\left(2 M_{S}+m_{l}\right) .
$$

The detuning between the $m_{l}=1$ and $m_{l}=-1$ states inside a single $M_{S}$ manifold is $2 \mu_{\mathrm{B}} B_{z}$. This has to be larger than the anisotropic matrix elements of dipole-dipole interactions, which for Lithium atoms and our typical parameters also makes it larger than the fine structure splitting. This implies we are in a strong field regime, where spin and angular momentum couple separately to the magnetic field, which effectively removes the fine structure and gives an energy level structure sketched in Fig. 11(b). The energy spacing in this 
strong field regime is $E_{\mathcal{M F}}=\mu_{\mathrm{B}} B_{z}$, which is of the order of $\sim 100 . .250 \mathrm{MHz}$.

The fine structure furthermore yields doublets for neighboring states with $\left(\Delta M_{S}, \Delta m_{l}\right)=( \pm 1, \mp 2)$, sketched as red and blue lines in Fig. 11(b). The only singlet states with $m_{l} \neq 0$ are the manifolds $\left(M_{S}, m_{l}\right)=(N / 2,1)$ and $\left(M_{S}, m_{l}\right)=(-N / 2,-$ $1)$. Hence we concentrate on the $\left(M_{S}, m_{l}\right)=(N / 2,1)$ manifold, which can be well addressed during the Rydberg excitation process. The magnetic field yields increasing decoupling of the $\left(M_{S}, m_{l}\right)=(N / 2,1)$ manifold from the $\left(M_{S}, m_{l}\right)=$ $(N / 2,-1)$ manifold with increasing magnetic field strength. We study this decoupling in detail, giving the Hamiltonian structure in Appendix AI and derive effective interactions in Appendix A2. The only coupling of the $\left(M_{S}, m_{l}\right)=(N / 2,1)$ state to other manifolds than $\left(M_{S}, m_{l}\right)=(N / 2,-1)$ is through spin-orbit interactions and can thus be neglected.

\section{Planar Rydberg aggregates in strong $B$ fields}

Here we derive the Hamiltonian for a Rydberg aggregate in an external magnetic field pointing in the $z$ direction, where the magnetic field shift is much larger than the fine structure. This strong field regime allows a reduction of the electronic Hilbert space to a single spin manifold. All atoms are assumed to be located within a plane, as in all cases considered here, with quantization axis perpendicular to that plane. Then, the $m_{l}=0$ manifold is already completely decoupled [11]. We are interested in $m_{l}=-1,1$ and thus neglect the $m_{l}=0$ manifold in the following. We use the aggregate states $\left|\pi_{k}\right\rangle$ as in the main article and denote with $|-1\rangle,|1\rangle$ the states of angular momentum magnetic quantum number $m_{l}=-1,1$. The combined basis is $\left|\pi_{k}, m_{l}\right\rangle=\left|\pi_{k}\right\rangle \otimes\left|m_{l}\right\rangle=\left|s \ldots\left(p, m_{l}\right) \ldots s\right\rangle$. We consider two Hilbert spaces: The "pure" aggregate space $V$, spanned by the basis $B[V]:=\left\{\left|\pi_{k}\right\rangle\right\}_{k=1}^{N}$ and the space including the angular momentum magnetic quantum numbers $\mathcal{V}$, spanned by $B[\mathcal{V}]:=\{|-1\rangle,|1\rangle\} \otimes B[V]$.

The dipole-dipole interaction Hamiltonian with magnetic field shift for fixed $m_{l}= \pm 1$ is given by

$$
\hat{H}_{m_{l}}(\mathbf{R}):=\hat{H}^{\mathrm{el}}(\mathbf{R})+m_{l} E_{\mathcal{M F}} \mathbb{1}[V],
$$

with $\hat{H}^{\mathrm{el}}(\mathbf{R})$ the Hamiltonian in (1) and $\mathbb{1}[V]$ the identity operator, acting on states of $V$.

The dipole-dipole transitions from $m_{l}=1$ to $m_{l}=-1$ are described by

$$
\hat{W}(\mathbf{R}):=\frac{d^{2}}{2} \sum_{p, q=1}^{N} R_{p q}^{-3} e^{-2 \mathrm{i} \phi_{p q}}\left|\pi_{p}\right\rangle\left\langle\pi_{q}\right|,
$$

where $d:=d_{v, 1 ; v, 0}$ is the radial dipole matrix element between $p$ and $s$ for principal quantum number $v$, and $R_{p q}=\left|\mathbf{R}_{p q}\right|$ and $\phi_{p q}$ are the modulus and azimuthal angle of the separation $\mathbf{R}_{p q}$ between atoms $p$ and $q$, within the coordinate system defining our quantization axes.

We treat now $\hat{H}_{m_{l}}(\mathbf{R})$ as unperturbed system and $\hat{W}(\mathbf{R})$ as perturbation and set up extended Hamiltonians:

$$
\begin{gathered}
\hat{\mathcal{H}}_{0}(\mathbf{R}):=\sum_{m_{l} \in\{-1,1\}}\left|m_{l}\right\rangle\left\langle m_{l}\right| \otimes \hat{H}_{m_{l}}(\mathbf{R}), \\
\hat{\mathcal{W}}(\mathbf{R}):=|1\rangle\langle-1|\otimes \hat{W}(\mathbf{R})+|-1\rangle\langle 1| \otimes \hat{W}^{\dagger}(\mathbf{R}),
\end{gathered}
$$

$$
\hat{\mathcal{H}}(\mathbf{R}):=\hat{\mathcal{H}}_{0}(\mathbf{R})+\hat{\mathcal{W}}(\mathbf{R}) .
$$

The Hamiltonian (A8) describes Rydberg aggregates with magnetic field shifts for the $m_{l}=-1,1$ states, but neglecting fine structure shifts.

\section{Effective interactions from block diagonalization}

To see how the mixing of the $m_{l}=-1,1$ manifolds due to the interaction described by $\hat{\mathcal{W}}(\mathbf{R})$ is weakened with increasing magnetic field, we block diagonalize $\hat{\mathcal{H}}(\mathbf{R})$, according to van Vleck perturbation theory [51] in a canonical form as outlined by Shavitt et al. [52]. We define the projectors,

$$
\hat{\mathcal{P}}_{m_{l}}:=\left|m_{l}\right\rangle\left\langle m_{l}\right| \otimes \mathbb{1}[V],
$$

and denote for every operator $\hat{\mathcal{A}}$ that acts on states in $\mathcal{V}$ the diagonal blocks with

$$
\hat{\mathcal{A}}_{D}=\hat{\mathcal{P}}_{-1} \hat{\mathcal{A}} \hat{\mathcal{P}}_{-1}+\hat{\mathcal{P}}_{1} \hat{\mathcal{A}} \hat{\mathcal{P}}_{1}
$$

and the off-diagonal blocks with

$$
\hat{\mathcal{A}}_{X}=\hat{\mathcal{P}}_{1} \hat{\mathcal{A}} \hat{\mathcal{P}}_{-1}+\hat{\mathcal{P}}_{-1} \hat{\mathcal{A}} \hat{\mathcal{P}}_{1}
$$

The goal is to find a unitary transformation, $\hat{\mathcal{U}}(\mathbf{R})$, such that $\mathcal{H}^{\prime}(\mathbf{R})=\hat{\mathcal{U}}^{\dagger}(\mathbf{R}) \hat{\mathcal{H}}(\mathbf{R}) \hat{\mathcal{U}}(\mathbf{R})$ is block diagonal, i.e., $\mathcal{H}_{X}^{\prime}(\mathbf{R})=0$. In the following we omit the dependency of the operators on $\mathbf{R}$ for better readability.

In canonical van Vleck perturbation theory, the transformation is rewritten as $\hat{\mathcal{U}}=\mathrm{e}^{\hat{\mathcal{G}}}$ with the property $\hat{\mathcal{G}}=-\hat{\mathcal{G}}^{\dagger}$ and $\hat{\mathcal{G}}_{D}=0$. These conditions lead to the following form of $\hat{\mathcal{G}}$ :

$$
\hat{\mathcal{G}}=|1\rangle\langle-1|\otimes \hat{G}-|-1\rangle\langle 1| \otimes \hat{G}^{\dagger},
$$

with $\hat{G}$ acting on states in $V$. An expansion in orders of the perturbation $\hat{\mathcal{W}}(\mathbf{R})$ [52] leads to equations for $\hat{\mathcal{G}}_{(i)}, i$ labeling different orders:

$$
\begin{gathered}
{\left[\hat{\mathcal{H}}_{0}, \hat{\mathcal{G}}_{(1)}\right]=-\hat{\mathcal{W}},} \\
{\left[\hat{\mathcal{H}}_{0}, \hat{\mathcal{G}}_{(2)}\right]=0,} \\
{\left[\hat{\mathcal{H}}_{0}, \hat{\mathcal{G}}_{(3)}\right]=-1 / 3\left[\left[\hat{\mathcal{W}}, \hat{\mathcal{G}}_{(1)}\right], \hat{\mathcal{G}}_{(1)}\right] .}
\end{gathered}
$$

Equations (A13)-(A15) already exploit that in our case $\hat{\mathcal{W}}_{D}=$ 0 . Using (A12) and (A6) to evaluate the left-hand side of these equations, we get

$$
\left[\hat{\mathcal{H}}_{0}, \hat{\mathcal{G}}_{(i)}\right]=|1\rangle\langle-1| \otimes\left(\left[\hat{H}, \hat{G}_{(i)}\right]+2 E_{\mathcal{M F}} \hat{G}_{(i)}\right)+\text { c.c.. }
$$

Evaluating also the right-hand side of (A13)-(A15), we obtain formulas for the $\hat{G}_{(i)}$ :

$$
\begin{gathered}
{\left[\hat{H}, \hat{G}_{(1)}\right]=-2 E_{\mathcal{M F}} \hat{G}_{(1)}-\hat{W},} \\
{\left[\hat{H}, \hat{G}_{(2)}\right]=-2 E_{\mathcal{M F}} \hat{G}_{(2)},} \\
{\left[\hat{H}, \hat{G}_{(3)}\right]=-2 E_{\mathcal{M F}} \hat{G}_{(3)}+\hat{F}\left(\hat{W}, \hat{G}_{(1)}\right),}
\end{gathered}
$$

where $\quad \hat{F}\left(\hat{W}, \hat{G}_{(1)}\right)=1 / 3\left(\hat{W}\left[\hat{G}_{(1)}^{\dagger}, \hat{G}_{(1)}\right]+\left\{\hat{W}, \hat{G}_{(1)} \hat{G}_{(1)}^{\dagger}\right\}+\right.$ $\left.2 \hat{G}_{(1)} \hat{W}^{\dagger} \hat{G}_{(1)}\right)$. The curly brackets denote the anticommutator. 
Equations (A17)-(A19) are of the form,

$$
\hat{X}=a[\hat{Y}, \hat{X}]+b \hat{Z},
$$

with constants $a=\left(-2 E_{\mathcal{M F}}\right)^{-1}, b=\left(\mp 2 E_{\mathcal{M F}}\right)^{-1}$, and operators $\hat{X}=\hat{G}_{(i)}, \hat{Y}=\hat{H}, \hat{Z} \in\{\hat{W}, 0, \hat{F}\}$. The solution to this equation is

$$
\hat{X}=b \sum_{k=0}^{\infty} a^{k}[\hat{Y}, \hat{Z}]_{k},
$$

with $[\hat{Y}, \hat{Z}]_{k}=\left[\hat{Y},[\hat{Y}, \hat{Z}]_{k-1}\right]$ and $[\hat{Y}, \hat{Z}]_{0}=\hat{Z}$. The expansion of the block diagonalization is given by

$$
\begin{gathered}
\hat{\mathcal{H}}_{(0)}^{\prime}=\hat{\mathcal{H}}_{0}, \\
\hat{\mathcal{H}}_{(1)}^{\prime}=0, \\
\hat{\mathcal{H}}_{(2)}^{\prime}=1 / 2\left[\hat{\mathcal{W}}, \hat{\mathcal{G}}_{(1)}\right], \\
\hat{\mathcal{H}}_{(3)}^{\prime}=1 / 2\left[\hat{\mathcal{W}}, \hat{\mathcal{G}}_{(2)}\right], \\
\hat{\mathcal{H}}_{(4)}^{\prime}=1 / 2\left[\hat{\mathcal{W}}, \hat{\mathcal{G}}_{(3)}\right]-1 / 24\left[\left[\left[\hat{\mathcal{W}}, \hat{\mathcal{G}}_{(1)}\right], \hat{\mathcal{G}}_{(1)}\right], \hat{\mathcal{G}}_{(1)}\right] .
\end{gathered}
$$

The first correction of the block-diagonalization procedure is (A24). Using solution (A21) for Eq. (A18), we see that $\hat{G}_{(2)}=0$ and so $\hat{\mathcal{H}}_{(3)}^{\prime}=0$. The second contribution is thus from $\hat{\mathcal{H}}_{(4)}^{\prime}$, the leading order of which is $\mathcal{O}\left(E_{\mathcal{M F}}^{-3}\right)$, thus we do not consider it further. We now concentrate on the Hamiltonian for states with $m_{l}=1, \hat{H}^{\prime}:=\left\langle 1\left|\hat{\mathcal{H}}^{\prime}\right| 1\right\rangle$. Using (A7), (A12), and (A21) in (A24), we can evaluate the corresponding first correction term,

$$
\hat{H}_{(2)}^{\prime}=\left(4 E_{\mathcal{M F}}\right)^{-1} \sum_{k=0}^{\infty}\left(-2 E_{\mathcal{M F}}\right)^{-k}[\hat{H}, \hat{W}]_{k} \hat{W}^{\dagger}+\text { c.c. }
$$

Let us define the maximum dipole-dipole interaction element of $\hat{H}, E_{\mathcal{D D}}^{\max }:=d^{2} /\left(6 R_{\min }^{3}\right)$, with $R_{\min }:=\min _{p, q: p \neq q} R_{p q}$. To assess if (A27) is small compared to $E_{\mathcal{D D}}^{\max }$, as required for $\hat{H}^{\prime} \approx \hat{H}_{m_{l}=1}$, we introduce rescaled quantities, $\hat{X}:=E_{\mathcal{D D}}^{\max } \hat{\tilde{X}}$, with $\hat{X} \in\left\{\hat{H}, \hat{H}^{\prime}, \hat{W}\right\}$. This yields $\left|\tilde{H}_{p q}\right| \leqslant 1$ and $\left|\tilde{W}_{p q}\right| \leqslant 3$. Introducing further a decoupling number, $\alpha:=E_{\mathcal{D D}}^{\max } /\left(2 E_{\mathcal{M F}}\right)$ and setting $E_{\mathcal{M F}}$ as our zero of energy, we find up to $\alpha^{2}$ the following effective interaction Hamiltonian for $m_{l}=+1$ :

$$
\hat{\tilde{H}}^{\prime} \approx \hat{\tilde{H}}+\alpha \hat{\tilde{W}} \hat{\tilde{W}}^{\dagger}+\alpha^{2} \hat{\tilde{W}} \hat{\tilde{H}} \hat{\tilde{W}}^{\dagger}-\frac{\alpha^{2}}{2}\left\{\hat{\tilde{H}}, \hat{\tilde{W}} \hat{\tilde{W}}^{\dagger}\right\} .
$$

Note that $\hat{\tilde{H}}^{\prime}$ is measured in units of $E_{\mathcal{D D}}^{\max }$. The leading order $\hat{\tilde{H}}$ of the effective Hamiltonian (A28) corresponds to our original isotropic model (1) and the additional terms can be explicitly constructed to allow for small anisotropic corrections.

\section{Comparison between isotropic, effective, and complete Hamiltonian}

In this section we assess how good the isotropic or the effective Hamiltonian (both without spin degrees of freedom) approximate the complete Hamiltonian, which includes spinorbit coupling in addition to the magnetic field.

We denote the space of the electron spin states of the $i$ th atom with $\mathcal{S}^{(i)}$, the basis of which is spanned by $B\left[\mathcal{S}^{(i)}\right]=$ $\left\{|-1 / 2\rangle_{(i)},|1 / 2\rangle_{(i)}\right\}$. The states $|-1 / 2\rangle_{(i)}$ denote downwards oriented electron spin and $|1 / 2\rangle_{(i)}$ upwards oriented electron spin for the $i$ th atom. The space of all $N$ electron spins is then given by $\mathcal{S}=\otimes_{i=1}^{N} \mathcal{S}^{(i)}$, with the product basis $B[\mathcal{S}]=\otimes_{i=1}^{N} B\left[\mathcal{S}^{(i)}\right]$. The spin-orbit interaction destroys the decoupling of the $m_{l}=0$ states, such that we have to redefine some quantities of Appendix A1. The space $\mathcal{V}$ is now spanned by $B[\mathcal{V}]:=\{|-1\rangle,|0\rangle,|1\rangle\} \otimes B[V]$, with $\left|m_{l}\right\rangle$ the states of the quantum number $m_{l} \in\{-1,0,1\}$. The Hamiltonian for the $m_{l}=0$ states is given by

$$
\hat{H}_{0}(\mathbf{R}):=-2 \hat{H}_{\mathrm{dd}}(\mathbf{R})+\hat{H}_{\mathrm{vdw}}(\mathbf{R}),
$$

with $\hat{H}_{\mathrm{dd}}(\mathbf{R})$ the resonant dipole-dipole Hamiltonian in (2a) and $\hat{H}_{\mathrm{vdw}}(\mathbf{R})$ the nonresonant van-der-Waals Hamiltonian in (2b). Note that $\hat{H}_{0}(\mathbf{R})$ experiences no magnetic field shift. We redefine $\hat{\mathcal{H}}_{0}(\mathbf{R})$ from (A6), such that it includes the $m_{l}=0$ states:

$$
\hat{\mathcal{H}}_{0}(\mathbf{R}):=\sum_{m_{l} \in\{-1,0,1\}}\left|m_{l}\right\rangle\left\langle m_{l}\right| \otimes \hat{H}_{m_{l}}(\mathbf{R}) .
$$

The Hamiltonian in (A8), $\hat{\mathcal{H}}(\mathbf{R})$, is now calculated with the redefined $\hat{\mathcal{H}}_{0}(\mathbf{R})$.

With these definitions, we can span the complete space $\mathfrak{V}:=\mathcal{V} \otimes \mathcal{S}$, describing the orientation of the $p$ states and the spins of the electrons together. The product basis, where spin and orbital angular momentum of the $p$ states are not combined to a total angular momentum, is then given by $B_{l s}[\mathfrak{V}]:=B[\mathcal{V}] \otimes B[\mathcal{S}]$.

The Hamiltonian $\mathcal{H}(\mathbf{R})$ in (A8) includes the magnetic field shifts for the orbital angular momentum only. The magnetic field shift for the spins is described by

$$
\mathcal{H}_{\mathcal{M F}-\mathcal{S}}:=E_{\mathcal{M F}} \sum_{\left|\mathbf{M}_{S}\right\rangle \in B[\mathcal{S}]} M_{S}\left(\mathbf{M}_{S}\right)\left|\mathbf{M}_{S}\right\rangle\left\langle\mathbf{M}_{S}\right| .
$$

The dipole-dipole interactions together with the total magnetic field shift is then given by

$$
\hat{\mathfrak{H}}_{\mathcal{D D}+\mathcal{M F}}(\mathbf{R}):=\mathcal{H}(\mathbf{R}) \otimes \mathcal{H}_{\mathcal{M F}-\mathcal{S}}
$$

To set up the spin-orbit interaction Hamiltonian in a simple way, it is useful to employ yet another basis. First we define the spin spaces $\mathcal{S}^{(\neq i)}$, which describe all spins except those of the the $i$ th atom, $\mathcal{S}^{(\neq i)}:=\otimes_{k \neq i}^{N} \mathcal{S}^{(k)}$. Their product basis $B\left[\mathcal{S}^{(\neq i)}\right]$ is spanned by $B\left[\mathcal{S}^{(\neq i)}\right]=\bigotimes_{k \neq i}^{N} B\left[\mathcal{S}^{(k)}\right]$. The spin-orbit coupling yields a total angular momentum, $\hat{\mathbf{J}}:=\hat{\mathbf{L}}+\hat{\mathbf{S}}$ per atom. The pair $\left(j, m_{j}\right)$ are the quantum numbers of $\hat{\mathbf{J}}$, with $j \in\{1 / 2,3 / 2\}$ and $m_{j} \in M_{j}:=\{-|j|$, $|j|+1, \ldots,|j|\}$. The result of the spin-orbit coupling is the fine-structure splitting $\Delta E_{\mathrm{FS}}$, between $p$ states with $j=3 / 2$ and $p$ states with $j=1 / 2$. To write down the spin-orbit Hamiltonian in its eigenbasis, we first introduce aggregate states which include the spin of the $p$ states, $\left|\pi_{k}, j, m_{j}\right\rangle:=$ $\left|s \ldots\left(p, j, m_{j}\right) \ldots s\right\rangle$. We now define spaces $\mathfrak{V}_{j} \subset \mathfrak{V}$, which we span with the basis $B\left[\mathfrak{V}_{j}\right]:=\otimes_{k=1}^{N}\left\{\left|\pi_{k}, j, m_{j}\right\rangle\right\}_{m_{j} \in M_{j}} \otimes$ $B\left[\mathcal{S}^{(\neq k)}\right]$. The orthogonal sum of both " $j$ spaces" spans the complete space, $\mathfrak{V}=\mathfrak{V}_{1 / 2} \oplus \mathfrak{V}_{3 / 2}$. This yields the 
eigenbasis of the spin-orbit Hamiltonian, $B_{j, m j}[\mathfrak{V}]:=$ $B\left[\mathfrak{V}_{1 / 2}\right] \cup B\left[\mathfrak{V}_{3 / 2}\right]$. Introducing the unitary transformation $\hat{\mathfrak{U}}$, which performs the basis transformation from $B_{j, m j}[\mathfrak{V}]$ to $B_{l s}[\mathfrak{V}]$, the spin-orbit Hamiltonian in the basis $B_{l s}[\mathfrak{V}]$ is given by

$$
\hat{\mathfrak{H}}_{\mathcal{S O}}=\Delta E_{F S} \hat{\mathfrak{U}}\left(\mathbb{O}\left[\mathfrak{V}_{1 / 2}\right] \oplus \mathbb{1}\left[\mathfrak{V}_{3 / 2}\right]\right) \hat{\mathfrak{U}}^{\dagger}
$$

where $\mathbb{O}\left[\mathfrak{V}_{1 / 2}\right]$ is the null operator acting on elements in $\mathfrak{V}_{1 / 2}$ and transforming them into its zero. Note that we thus shift the origin of energy to the $j=1 / 2$ manifold. The complete Hamiltonian is given by

$$
\hat{\mathfrak{H}}(\mathbf{R}):=\hat{\mathfrak{H}}_{\mathcal{D} D+\mathcal{M F}}(\mathbf{R})+\hat{\mathfrak{H}}_{\mathcal{S O}}
$$

We compare the three different Hamiltonians in (1), (A28) and (A34) by using them to calculate the eigenenergies for a four-atom system with a symmetric configuration $(p=0)$, as sketched in Fig. 1(a). We show for (A34) only the $\left(M_{S}, m_{l}\right)=$ $(N / 2,1)$ manifold, which is the one, we propose to work with. The positions of atoms $(2,3)$ are fixed, whereas the positions of atoms 0 and 1 are parametrized as $x_{0}=-x$ and $x_{1}=a_{1}+x$. The eigenenergies of all three Hamiltonians are plotted as a function of the coordinate $x$ in Fig. 12. The isotropic model in (1) approximates (A34) well for all locations crucial in our simulations, as shown in Figs. 12(a)-12(c). Crucial for the simulations are configurations with small $x$ values, where the atoms are accelerated due to the interactions, and in the neighborhood of the conical intersection. The agreement is not good for the equidistant linear trimer configuration $x=d$. The excitation is there mostly delocalized and the phase of the dipole-dipole interaction plays a role. The effective Hamiltonian in (A28) approximates the complete one for this configuration very well, as shown in Figs. 12(d)-12(g). It appears that the Hamiltonian of order $\alpha$ in (A28) approximates (A34) better than the order $\alpha^{2}$ version. This may be since (A28) does not take the spin-orbit coupling into account and the fine structure is of the order of the $\alpha^{2}$ corrections. A better description beyond the $\alpha$ correction would then require a block diagonalization, which explicitly includes spin-orbit coupling.

As expected increasing the magnetic field strength improves the decoupling of the $\left(M_{S}, m_{l}\right)=(N / 2,1)$ manifold. This
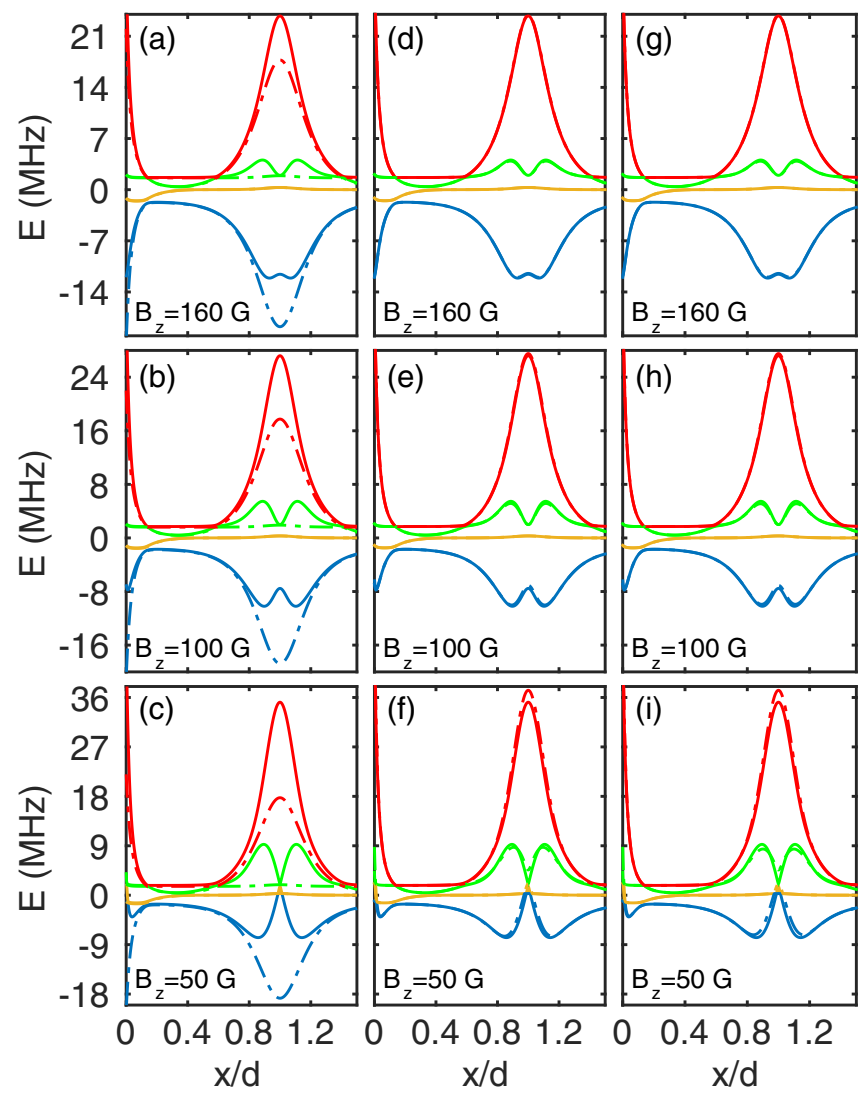

FIG. 12. Comparison of energy spectra from different approximations of the complete Hamiltionian, for a double dimer as sketched in Fig. 1 (a). We use $v=80$, which yields a fine-structure splitting of $\Delta E_{\mathrm{FS}}=0.15 \mathrm{MHz}$ [53]. Further parameters are $p=0, d=40 \mu \mathrm{m}$, $a_{1}=8 \mu \mathrm{m}$, and $a_{2}=19 \mu \mathrm{m}$. Atoms 2 and 3 are fixed, whereas the positions of atoms 0 and 1 are parametrized, such that $x_{0}=-x$ for atom 0 and $x_{1}=a_{1}+x$ for atom 1 . We compare the complete model (solid lines) with different approximate models (dashed dotted lines) in different columns. The approximate models are as follows: (a)-(c) purely isotropic model used for the main results in this paper, with Hamiltonian given in (1); (d)-(f) corrected model with effective Hamiltonian (A28) up to first order in $\alpha$; (g)-(i) same as (d)-(f), but using (A28) up to second order in $\alpha$. We consider three different magnetic field strengths: $B_{z}=50 \mathrm{G}$ in the lower row, $B_{z}=100 \mathrm{G}$ in the adjacent row, and $B_{z}=160 \mathrm{G}$ in the upper row. The energy of infinitely separated atoms is set to zero.

results in a better agreement between the reduced models and the complete model for higher field strengths.
[1] M. D. Lukin, M. Fleischhauer, R.Côté, L. M. Duan, D. Jaksch, J. I. Cirac, and P. Zoller, Phys. Rev. Lett. 87, 037901 (2001).

[2] E. Urban, T. A. Johnson, T. Henage, L. Isenhower, D. D. Yavuz, T. G. Walker, and M. Saffman, Nature Physics 5, 110 (2009).

[3] A. Gaëtan, Y. Miroshnychenko, T. Wilk, A. Chotia, M. Viteau, D. Comparat, P. Pillet, A. Browaeys, and P. Grangier, Nature Physics 5, 115 (2009).
[4] D. Tong, S. M. Farooqi, J. Stanojevic, S. Krishnan, Y. P. Zhang, R. Côté, E. E. Eyler, and P. L. Gould, Phys. Rev. Lett. 93, 063001 (2004).

[5] C. Ates, T. Pohl, T. Pattard, and J. M. Rost, Phys. Rev. A 76, 013413 (2007).

[6] K. Singer, M. Reetz-Lamour, T. Amthor, L. G. Marcassa, and M. Weidemüller, Phys. Rev. Lett. 93, 163001 (2004).

[7] C. H. Greene, A. S. Dickinson, and H. R. Sadeghpour, Phys. Rev. Lett. 85, 2458 (2000). 
[8] I. C. H. Liu, J. Stanojevic, and J. M. Rost, Phys. Rev. Lett. 102, 173001 (2009).

[9] C. Ates, A. Eisfeld, and J. M. Rost, New J. Phys. 10, 045030 (2008).

[10] S. Wüster, C. Ates, A. Eisfeld, and J. M. Rost, Phys. Rev. Lett. 105, 053004 (2010).

[11] S. Möbius, S. Wüster, C. Ates, A. Eisfeld, and J. M. Rost, J. Phys. B: At. Mol. Opt. Phys. 44, 184011 (2011).

[12] S. Wüster, A. Eisfeld, and J. M. Rost, Phys. Rev. Lett. 106, 153002 (2011).

[13] H. Zoubi, A. Eisfeld, and S. Wüster, Phys. Rev. A 89, 053426 (2014).

[14] S. Möbius, M. Genkin, S. Wüster, A. Eisfeld, and J.-M. Rost, Phys. Rev. A 88, 012716 (2013).

[15] D. Barredo, H. Labuhn, S. Ravets, T. Lahaye, A. Browaeys, and C. S. Adams, Phys. Rev. Lett. 114, 113002 (2015).

[16] G. Günter, H. Schempp, M. Robert-de-Saint-Vincent, V. Gavryusev, S. Helmrich, C. S. Hofmann, S. Whitlock, and M. Weidemüller, Science 342, 954 (2013).

[17] S. Bettelli, D. Maxwell, T. Fernholz, C. S. Adams, I. Lesanovsky, and C. Ates, Phys. Rev. A 88, 043436 (2013).

[18] O. Mülken, A. Blumen, T. Amthor, C. Giese, M. ReetzLamour, and M. Weidemüller, Phys. Rev. Lett. 99, 090601 (2007).

[19] H. Park, P. J. Tanner, B. J. Claessens, E. S. Shuman, and T. F. Gallagher, Phys. Rev. A 84, 022704 (2011).

[20] H. Park, E. S. Shuman, and T. F. Gallagher, Phys. Rev. A 84, 052708 (2011).

[21] W. Li, P. J. Tanner, and T. F. Gallagher, Phys. Rev. Lett. 94, 173001 (2005).

[22] S. Westermann, T. Amthor, A. de Oliveira, J. Deiglmayr, M. Reetz-Lamour, and M. Weidemüller, Eur. Phys. J. D 40, 37 (2006).

[23] S. Ravets, H. Labuhn, D. Barredo, L. Béguin, T. Lahaye, and A. Browaeys, Nature Physics 10, 914 (2014).

[24] M. Saffman, T. G. Walker, and K. Mølmer, Rev. Mod. Phys. 82, 2313 (2010).

[25] H. Weimer, M. Müller, I. Lesanovsky, P. Zoller, and H. P. Büchler, Nature Phys. 6, 382 (2010).

[26] H. Schempp, G. Günter, S. Wüster, M. Weidemüller, and S. Whitlock, Phys. Rev. Lett. 115, 093002 (2015).

[27] D. W. Schönleber, A. Eisfeld, M. Genkin, S. Whitlock, and S. Wüster, Phys. Rev. Lett. 114, 123005 (2015).

[28] J. Frenkel, Phys. Rev. 37, 17 (1931).

[29] M. Dantus and A. Zewail, Chem. Rev. 104, 1717 (2004).

[30] S. Perun, A. L. Sobolewski, and W. Domcke, Chem. Phys. 313, 107 (2005).

[31] K. Leonhardt, S. Wüster, and J. M. Rost, Phys. Rev. Lett. 113, 223001 (2014).

[32] L. Li, Y. O. Dudin, and A. Kuzmich, Nature (London) 498, 466 (2013).

[33] R. Mukherjee, J. Millen, R. Nath, M. P. A. Jones, and T. Pohl, J. Phys. B: At. Mol. Opt. Phys. 44, 184010 (2011).
[34] T. F. Gallagher, Rydberg Atoms (Cambridge University Press, Cambridge, 1994).

[35] J. C. Tully and R. K. Preston, J. Chem. Phys. 55, 562 (1971).

[36] S. Hammes-Schiffer and J. C. Tully, J. Chem. Phys. 101, 4657 (1994).

[37] M. Barbatti, Wiley Interdisciplinary Reviews-Computational Molecular Science 1, 620 (2011).

[38] Note that $\left|\varphi_{\text {adj }}\right\rangle$ is called $\left|\varphi_{\text {mid }}\right\rangle$ in Ref. [31].

[39] Note that in the energy spectra for the four- (seven-) atom case, there is one crossing (are three crossings) of eigenenergies already before the wave-packet splitting takes place. These are formally also CIs. However, they simply reflect the fact that two eigenvalues of excitons localized on spatially far separated sites cross. These would also cross if our two chains were not coupled. Kinematically, the velocity $\dot{\mathbf{R}}$ is constrained such that these earlier CIs are always exactly hit, causing a diabatic transition between surfaces. Also in our simulations, all trajectories safely jump to the correct surface at these points.

[40] This counterintuitive fact is due to the excitation distribution among the three atoms on the middle energy surface. Here the excitation resides predominantly on the atom farthest away, since forces are determined by a combination of excitation probability and relative distance, in this case this farthest atom experiences the largest force.

[41] R. Celistrino Teixeira, C. Hermann-Avigliano, T. L. Nguyen, T. Cantat-Moltrecht, J. M. Raimond, S. Haroche, S. Gleyzes, and M. Brune, arXiv:1502.04179.

[42] For a formal definition of the atomic density, see the supplemental information of [31].

[43] The purity is defined by $P=\operatorname{Tr}\left[\hat{\sigma}^{2}\right]$, where $\hat{\sigma}=$ $\sum_{n, m} \sigma_{n, m}\left|\pi_{n}\right\rangle\left\langle\pi_{m}\right|$ is the reduced electronic density matrix, which describes the electronic state of the system, after tracing over the atomic positions. The matrix elements are given by $\sigma_{n m}=\left\langle c_{n}^{*} c_{m}\right\rangle_{T}$ for the surface hopping method. The brackets denote the trajectory average.

[44] F. Robicheaux, J. V. Hernandez, T. Topcu, and L. D. Noordam, Phys. Rev. A 70, 042703 (2004).

[45] K. Leonhardt, S. Wüster, and J. M. Rost, arXiv:1602.01032.

[46] T. Breeden and H. Metcalf, Phys. Rev. Lett. 47, 1726 (1981).

[47] S. Wüster, C. Ates, A. Eisfeld, and J. M. Rost, New J. Phys. 13, 073044 (2011).

[48] M. Genkin, S. Wüster, S. Möbius, A. Eisfeld, and J. M. Rost, J. Phys. B: At. Mol. Opt. Phys. 47, 095003 (2014).

[49] S. Möbius, M. Genkin, A. Eisfeld, S. Wüster, and J.-M. Rost, Phys. Rev. A 87, 051602(R) (2013).

[50] S. Wüster, S. Möbius, M. Genkin, A. Eisfeld, and J.-M. Rost, Phys. Rev. A 88, 063644 (2013).

[51] J. H. Van Vleck, Phys. Rev. 33, 467 (1929).

[52] I. Shavitt and L. T. Redmon, J. Chem. Phys. 73, 5711 (1980).

[53] P. Goy, J. Liang, M. Gross, and S. Haroche, Phys. Rev. A 34, 2889 (1986). 